\title{
A test of the regulatory fit hypothesis in perceptual classification learning
}

\author{
W. TODD MADDOX, GRANT C. BALDWIN, and ARTHUR B. MARKMAN \\ University of Texas, Austin, Texas
}

\begin{abstract}
This article builds a bridge between research on regulatory focus in motivation and classification learning. It tests the hypothesis that a fit between the situational regulatory focus and the reward structure of the task leads to greater cognitive flexibility than does a mismatch between situational focus and the reward structure and that the fit between the regulatory-focus-induced processing characteristics and the nature of the environment influences performance. In Experiment 1, we used a classification task for which cognitive flexibility should be advantageous and examined both gains (Experiment 1A) and losses (Experiment 1B) reward structures. In Experiments 2 and 3, we used a classification task for which cognitive flexibility should be disadvantageous. In Experiment 2, we used a gains reward structure, and in Experiment 3, we used a losses reward structure. As was predicted, when cognitive flexibility was advantageous, the participants in a regulatory fit showed faster learning and more quickly shifted toward the optimal response strategy. Also as was predicted, when cognitive flexibility was disadvantageous, the participants in a regulatory mismatch showed faster learning and more quickly shifted toward the optimal response strategy. Implications for current theories of motivation and classification learning are discussed.
\end{abstract}

Nearly all human behavior has some motivational component. We learn and select behaviors on the basis of the information currently present in the environment, but also on the basis of our current motivational state, in order to approach positive outcomes or avoid negative outcomes. Despite the fact that learning and motivation are intimately related behaviorally, they are artificially separated within psychology. Research in cognitive psychology has typically focused on the information that people process, with little regard for the factors that drive people to act, whereas motivation is generally studied in social and educational psychology. A major aim of this research is to bring motivation back into the realm of cognitive research.

Much recent work has explored the influence of active goals on behavior (e.g., Aarts, Gollwitzer, \& Hassin, 2004; Ferguson \& Bargh, 2004; Fishbach, Friedman, \& Kruglanski, 2003; Higgins, 2000). Furthermore, the motivational system has been the target of theories in cognitive neuroscience (e.g., Ashby, Isen, \& Turken, 1999; Gray, 1982). Despite the clear interest in the influence of motivation on cognition, there has been little cognitive research that

This research was supported in part by National Institutes of Health Grants R01 MH59196 to W.T.M., R21 DA15211 to A.B.M., and AFOSR FA9550-06-1-0204 to W.T.M. and A.B.M. We thank Greg Ashby, Richard Ivry, and Alan Pickering for useful discussions that influenced this work and William von Hippel and three anonymous reviewers for comments on an earlier version of the manuscript. We thank Scott Lauritzen for help with data collection. Correspondence concerning this article should be addressed to W. T. Maddox or A. B. Markman, Department of Psychology, University of Texas, 1 University Station A8000, Austin, TX 78712 (e-mail: maddox@psy.utexas.edu or markman@psy.utexas.edu). has targeted the effects of motivation on learning, and to our knowledge, none of this work has used tasks that are understood well enough that computational modeling approaches can be used to rigorously examine the effects of motivation on learning processes (see Maddox, Markman, \& Baldwin, 2006; Markman, Baldwin, \& Maddox, 2005). As our learning framework, we will focus on the domain of perceptual classification learning. Perceptual classification learning offers a nice starting point for examining the motivation-learning interface, because quick and accurate classification is central to the survival of all organisms and is performed thousands of times a day. Also of importance, sophisticated mathematical models have been developed that provide insights into the strategies that people adopt throughout learning. These models support fine-grained analyses of the performance of individual participants. In this article, we will examine the influence of motivational factors on perceptual classification learning by incorporating ideas developed in Higgins's regulatory focus theory (Higgins, 1987, 1997) and his more recent notion of regulatory fit (Higgins, 2000; Higgins, Chen Idson, Freitas, Spiegel, \& Molden, 2003) into the perceptual classification learning task.

In the next section, we will provide a brief overview of regulatory focus theory (Higgins, 1987, 1997) and the notion of regulatory fit (Higgins, 2000; Higgins et al., 2003), reviewing some of the relatively straightforward experimental manipulations that have been used to influence participants' motivational states. Then, we will discuss the implications of regulatory fit for perceptual classification and will outline a series of predictions. Next, we will present data from three experiments in which these predic- 
tions were tested by exploring the influence of regulatory fit on people's flexibility in classification. Finally, we will discuss the implications of these findings for behavioral research.

\section{Regulatory Focus Theory and the Notion of Regulatory Fit}

The motivation literature makes a distinction between approach and avoidance goals (e.g., Carver \& Scheier, 1998; Lewin, 1935; Markman \& Brendl, 1999; Miller, 1959). Approach goals have desirable states that someone wishes to bring about. Avoidance goals have undesirable end states that someone wishes to prevent from occurring. Higgins and colleagues (Higgins, 1987, 1997) extended this idea of approach and avoidance to suggest that orthogonal to approach and avoidance goals, there may be psychological states of readiness or sensitivity for potential gain/nongain or nonloss/loss goals that tune the sensitivity of the motivational system. On this view, a promotion focus activates an approach mode of processing that focuses the motivational system on the presence or absence of gains in the environment. In contrast, a prevention focus activates an avoidance mode of processing that focuses the motivational system on the presence or absence of losses in the environment. ${ }^{1}$ Higgins and colleagues demonstrated that the type of regulatory focus people have in a given task affects cognitive processing and decision making (Crowe \& Higgins, 1997; Shah \& Higgins, 1997).

An advantage of using regulatory focus theory to study the effects of motivation on cognition is the fact that situational manipulations of regulatory focus are quite easy to instantiate but can lead to powerful effects. The basic logic of these manipulations is to focus people on global gains/nongains for a situational promotion focus or global nonlosses/losses for a situational prevention focus. The manipulation need not even be directly connected to the task of central interest in the study. For example, Crowe and Higgins (1997) told all participants that they would complete a recognition memory task, followed by a second task. The promotion participants were told that if they did well on the recognition task, the second task would be one that they "liked," whereas the prevention participants were told that if they did not do well on the recognition task, the second task would be one that they "disliked" (liked and disliked tasks were determined separately for each participant on the basis of pretesting). A different manipulation was used by Lee and Aaker (2004). They exposed participants to advertisements and varied the tag line in the ad so that it focused either on potential positive states (e.g., "get energized") for a promotion focus or on potential negative states (e.g., "prevent clogged arteries") for a prevention focus. As a third example, Shah, Higgins, and Friedman (1998) had participants solve anagrams. The promotion participants were paid a base salary of $\$ 4$ but were told that they could earn an extra $\$ 1$ if they found $90 \%$ of the anagram solutions, whereas the prevention participants were paid a base salary of $\$ 5$ but were told that they would lose $\$ 1$ if they missed more than $10 \%$ of the anagram solutions. In all cases, this simple, easy- to-instantiate situational regulatory focus manipulation had a large effect on performance. To anticipate, we used a situational regulatory focus manipulation modeled after that in Shah et al. in the experiments reported below.

Higgins and colleagues extended the notion of regulatory focus by suggesting that the influence of a person's situational regulatory focus (promotion or prevention) on cognition depends on the regulatory fit between that global situational focus and the more local gains and losses available in the task being performed (Avnet \& Higgins, 2003; Higgins, 2000; Higgins et al., 2003; Lee \& Aaker, 2004; Shah et al., 1998). We will refer to the gains and losses associated with the task as the reward structure of the task. Their research suggests that a regulatory fit between the situational regulatory focus and the reward structure of the task (i.e., a situational promotion focus paired with a gains reward structure or a situational prevention focus paired with a losses reward structure) leads to a feeling of fluency that influences cognition and evaluation. A regulatory mismatch between the situational regulatory focus and the reward structure of the tasks (i.e., a situational promotion focus paired with a losses reward structure or a situational prevention focus paired with a gains reward structure) reduces this feeling of fluency. Shah et al. hypothesized that participants given a situational promotion focus should pursue the gaining of points more than the losing of points and that participants given a situational prevention focus should pursue avoiding the losing of points more than the gaining of points. As a test of this hypothesis, the participants were asked to solve 6 "red" and 6 "green" anagrams and were told that the goal was to finish with at least 4 points. The participants were told that for each green anagram, they would earn 1 point if they found all of the words (a gains reward structure). The participants were also told that for each red anagram they would lose 1 point if they did not find all of the words (a losses reward structure). The situational promotion focus participants were told that they would earn $\$ 4$ but that if they gained 4 or more points, they would earn an extra $\$ 1$. The situational prevention focus participants were told that they would earn $\$ 5$ but that if they failed to gain 4 or more points, they would lose $\$ 1$. As was predicted, the participants given a situational promotion focus pursued the green anagrams (the gains reward structure) more than the red (the losses reward structure), whereas the participants given a situational prevention focus pursued the red anagrams more than the green.

We expanded on this regulatory fit hypothesis by suggesting that regulatory fit actually increases people's cognitive flexibility, relative to a regulatory mismatch. On this view, flexibility is defined as an increase in an individual's ability or willingness to try different strategies across trials to achieve some stated goal, as opposed to utilizing gradual incremental changes in strategy. Importantly, cognitive flexibility is not always advantageous for efficient learning, and thus, a regulatory fit is not always advantageous. We will turn now to a brief review of our regulatory fit hypothesis as it applies to perceptual classification learning. 


\section{Regulatory Fit, Cognitive Flexibility, and Perceptual Classification Learning}

Classification learning involves laying down a memory trace that leads to faster, more accurate responding in the future. It is generally agreed that mammals have multiple memory systems (Poldrack \& Packard, 2003; Schacter, 1987; Squire, 1992), and there is an overwhelming body of evidence to suggest that multiple classification learning systems exist (for reviews, see Ashby \& Maddox, 2005; Erickson \& Kruschke, 1998; Kéri, 2003; Maddox \& Ashby, 2004).

One multiple systems model that has stimulated much research is the competition between verbal and implicit systems model (COVIS; Ashby, Alfonso-Reese, Turken, \& Waldron, 1998; Ashby \& Waldron, 1999). This model postulates two systems that compete throughout learning: an explicit, hypothesis-testing system and an implicit, procedural-based learning system. Importantly, the model assumes that people have a bias toward the hypothesistesting system early in learning. COVIS assumes that rule-based category learning is dominated by the explicit hypothesis-testing system, whereas information integration category learning is dominated by the implicit procedural-based learning system.

With rule-based categories, the rule that maximizes accuracy is usually easy to describe verbally. For example, Figure 1A presents a scatterplot of stimuli from a twocategory rule-based condition with simple line stimuli that vary across trials in length, orientation, and horizontal position. The optimal decision rule is conjunctive and requires the participant to set a criterion on length and a criterion on orientation and to use the following rule: Respond A if the length is long and the orientation is steep; otherwise, respond B. A participant using the optimal length and orientation decision criteria could attain $100 \%$ accuracy. The position dimension is irrelevant, but the best unidimensional rule for position yields $83 \%$ accuracy. (A second rule-based category structure is presented in Figure 1B and will be described later.)

Figure 1C presents a scatterplot of stimuli from a twocategory information integration condition with the same three-dimensional stimuli as those used in Figure 1A, along with the optimal diagonal decision rule. Again, position is irrelevant but can yield $83 \%$ correct, whereas the optimal decision rule requires an integration of the length and orientation information and can yield $100 \%$ accuracy. Because length and orientation are measured in different units and because the decision rule is not parallel to the coordinate axes, it cannot be described verbally. It is important to note that there is a large body of evidence to support the existence of the hypothesis-testing and procedural-based learning systems. This work suggests that the cognitive processes, as well as the relevant underlying neurobiology, differ across the two systems. A detailed review is beyond the scope of this article (see Ashby \& Maddox, 2005; Ashby $\&$ Valentin, 2005; Maddox \& Ashby, 2004). With respect to the notion that a regulatory fit leads to cognitive flexibility, what is most important is that the hypothesis-testing system relies predominantly on frontal brain regions known

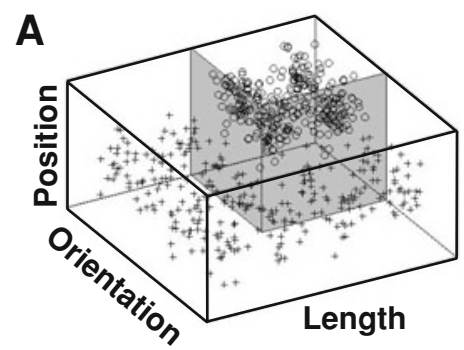

B
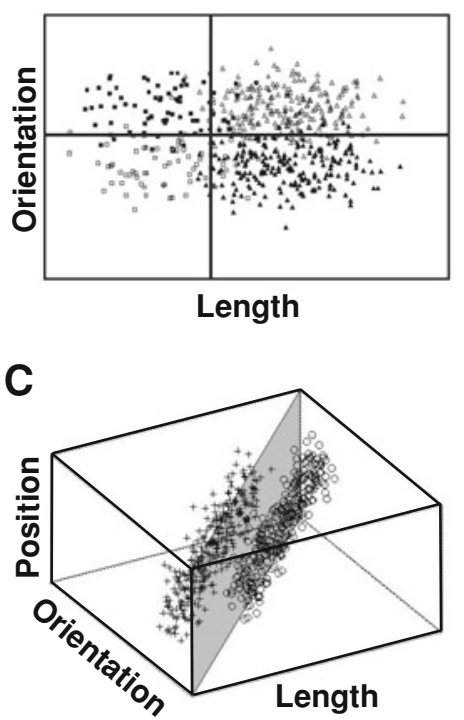

Figure 1. (A) The two-category conjunctive-rule-based category structure. (B) The four-category conjunctive-rule-based category structure, along with optimal decision bounds. (C) A twocategory information integration category structure. In panels $A$ and $C$, plus signs (+) denote Category $A$, open circles $(O)$ denote Category $B$, and the gray planes represent the optimal decision bound. In panel $B$, open squares denote Category $A$ stimuli, filled squares denote Category $\mathbf{B}$, filled triangles denote Category $\mathbf{C}$, and open triangles denote Category $D$.

to be involved in flexible cognitive processing, whereas the procedural-based learning system relies predominantly on subcortical regions, such as the tail of the caudate nucleus.

In this research we tested the hypothesis that a regulatory fit affects information processing by increasing cognitive flexibility, whereas a regulatory mismatch reduces cognitive flexibility. Cognitive flexibility implies an increase in one's ability or willingness to try different strategies across trials to achieve some stated goal, as opposed to utilizing more gradual incremental changes in strategy. When cognitive flexibility is advantageous for solving the task, a person in a regulatory fit should perform better than a person in a regulatory mismatch. On the other hand, when cognitive flexibility is disadvantageous and, instead, a gradual incremental approach is advantageous for solving the task, a person in a regulatory mismatch should perform better than a person in a regulatory fit.

There are a number of reasons to think that this hypothesis is plausible. First, Crowe and Higgins (1997; Shah 
et al., 1998) found that a regulatory fit led participants to generate more alternatives in an anagram task. Solving anagrams requires the participant to select and test numerous alternatives and, thus, can be defined generally as involving cognitive flexibility. Second, in a recent study (Markman, Maddox, Worthy, \& Baldwin, in press), we found that a regulatory fit led participants to more associates in the remote associates task (RAT; Mednick, Mednick, \& Mednick, 1964), in which people find a word that is associated to three test words. The RAT is a classic measure of cognitive flexibility. Finally, there is growing neuroscientific evidence linking regulatory focus to activation in frontal brain areas. For example, Amodio, Shah, Sigelman, Brazy, and Harmon-Jones (2004; see also Cunningham, Raye, \& Johnson, 2005) used ERPs to demonstrate a relationship between regulatory focus and activation in frontal brain areas. Although clearly more work is needed to fully understand the neurobiological underpinnings of cognitive flexibility, it seems reasonable to assume the involvement of frontal areas.

To test the hypothesis that regulatory fit affects classification learning through its effects on cognitive flexibility, we need a classification-learning task for which variations in cognitive flexibility will lead to observable performance differences. The proposed link between regulatory fit, cognitive flexibility, and the involvement of frontal brain regions suggests that regulatory fit manipulations should have the strongest effects on rule-based classification learning (Maddox et al., 2006). Consider the rulebased classification-learning task outlined in Figure 1A. The optimal rule requires the participant to respond " $\mathrm{A}$ " to long, steep angle lines, to respond "B" to all other lines, and to ignore position, even though pilot studies collected in our lab suggest that the position dimension is highly salient and that participants focus initially on this dimension. In this case, cognitive flexibility would be advantageous, because finding the optimal rule would require the participant to be willing to abandon the highly salient position dimension in favor of a lower salience, conjunctive rule for length and orientation. Thus, we predicted that participants asked to learn the Figure 1A category structure should be more likely to learn the task if they were in a regulatory fit than if they were in a regulatory mismatch. Experiment 1A tested this prediction, using a gains reward structure in which participants were trying to maximize the number of points earned. Experiment 1B tested this prediction using a losses reward structure in which participants were trying to minimize the number of points lost.

The regulatory fit predictions for Experiment 1 are summarized in Table 1. In short, the participants in a regulatory fit (e.g., situational promotion with a gains reward structure and situational prevention with a losses reward structure) were expected to do better than participants in a regulatory mismatch (e.g., situational promotion with a losses reward structure and situational prevention with a gains reward structure). A complete test of the regulatory fit hypothesis required that we also examine classificationlearning situations in which cognitive flexibility was dis-
Table 1

Perceptual Classification Learning Predictions From the Regulatory Fit Hypothesis

\begin{tabular}{lcr}
\hline \multirow{2}{*}{$\begin{array}{c}\text { Situational } \\
\text { Focus }\end{array}$} & \multicolumn{2}{c}{ Reward Structure of the Task } \\
\cline { 2 - 3 } & Gains & Losses \\
\hline $\begin{array}{l}\text { Experiment 1: Flexibility Advantageous } \\
\text { Promotion }\end{array}$ & better & \\
Prevention & worse & worse \\
Experiments 2 and 3: Flexibility Disadvantageous & better \\
Promotion & worse & \\
Prevention & better & better \\
& & worse \\
\hline
\end{tabular}

advantageous. When the nature of the optimal categorization rule was obvious from the outset and the participant needed only fine-tune the decision criteria, heightened cognitive flexibility might lead to too much variability in strategy use and, thus, poorer performance (assuming that the rule was difficult enough that the participants did not achieve optimal performance quite quickly regardless of their willingness to be flexible). In this case, we should observe better performance by those with a regulatory mismatch than by those with a regulatory fit.

Experiment 2 tested the hypothesis that a regulatory mismatch would yield better performance in a rule-based task for which the optimal rule was obvious from the outset. Experiment 2 focused on a gains reward structure and used the four-category rule-based problem displayed in Figure 1B. The stimulus was a line whose length and orientation varied across trials, and the optimal rule was to "respond $\mathrm{A}$ if the length is short and the orientation is shallow; respond B if the length is short and the orientation is steep; respond $\mathrm{C}$ if the length is long and the orientation is shallow; and respond $\mathrm{D}$ if the length is long and the orientation is steep." The type of cognitive flexibility associated with qualitative shifts in the nature of the decision rule would not be useful here. Instead, good performance would be achieved through slower, more incremental changes in the decision criterion values that are associated with lower levels of cognitive flexibility. Thus, we predicted that the participants in a situational prevention focus (i.e., those in a regulatory mismatch) would show better learning than would the participants in a situational promotion focus (see Table 1).

In Experiment 3, we examined a category structure for which cognitive flexibility was disadvantageous and used a losses reward structure. For reasons that will be outlined later, we abandoned the four-category rule-based task in favor of the two-category information integration task displayed in Figure 1C that shared many properties with the Figure 1A category structure. On the surface, the regulatory-fit-cognitive-flexibility hypothesis seems to predict little if any effect on information integration category learning, because information integration category learning is mediated by a procedural-based learning system that does not utilize frontal brain regions associated with cognitive flexibility. Recall, though, that COVIS assumes that there is a bias toward the hypothesis-testing system early in learning (Ashby et al., 1998). One reason- 
able prediction is that a regulatory fit will lead to worse information integration performance early in learning because of an increased reliance on hypothesis-testing strategies. Thus, we predicted that the participants in a situational promotion focus would show better learning early in the task than would the participants in a situational prevention focus. Note that this is the opposite of the prediction for Experiment 2 but is in line with the regulatory fit hypothesis (see Table 1).

In all three experiments, we examined the effects of regulatory fit on learning by using global measures of accuracy. In addition, and to better understand the nature of the participants' strategy development, we also applied a series of computational models to the data.

\section{EXPERIMENT 1}

Experiment 1 provided a critical test of the predicted interaction between situational regulatory focus and the reward structure of the task on performance in a perceptual classification task for which cognitive flexibility was advantageous. The stimulus on each trial was a line whose length, orientation, and horizontal position on the computer screen varied across trials. The category structure is displayed in Figure 1A. The optimal decision rule was

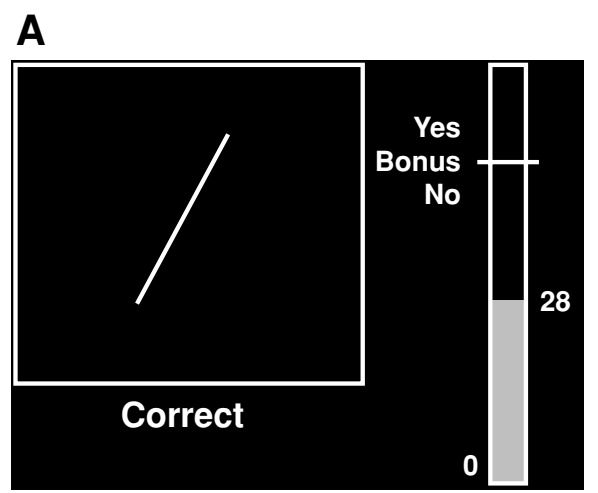

B

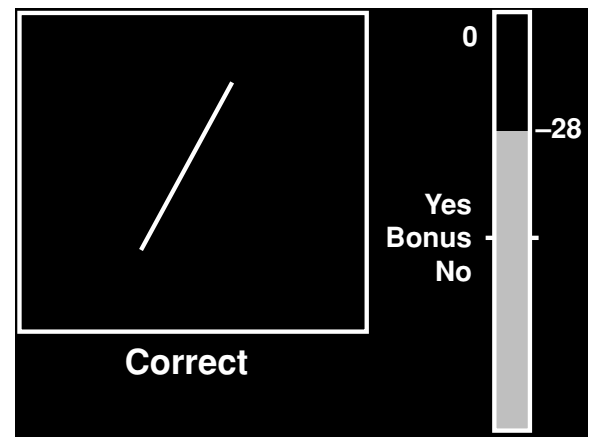

Figure 2. (A) Mock-up of experiment screen from the gains version of the task. The point meter starts at the bottom of the screen and moves toward the top of the screen. (B) Mock-up of experiment screen from the losses version of the task. The point meter starts at the top of the screen and moves toward the bottom of the screen. conjunctive and required the participant to set a criterion on length and a criterion on orientation and to use the following rule: Respond $\mathrm{A}$ if the length is long and the orientation is steep; otherwise, respond B. A participant using the optimal rule could attain $100 \%$ accuracy, whereas the most accurate unidimensional rules for length, orientation, and position yielded $83 \%$ accuracy. Each participant completed twelve 48-trial blocks in the experiment.

In Experiment 1A, a gains reward structure was used in which the participant's goal was to maximize points earned and to exceed a performance criterion. In Experiment 1B, a losses reward structure was used in which the participants' goal was to minimize the number of points lost. At the beginning of each block, a thermometer scale was set to zero. The thermometer scale allowed the participants to track their cumulative point total, relative to the performance criterion. A sample screen from the gains reward structure is displayed in Figure 2A, and a sample screen from the losses reward structure is displayed in Figure 2B.

The participants in the situational promotion focus condition were told that they could earn an entry into a drawing for $\$ 50$ if they exceeded a performance criterion during the final block of trials (equivalent to meeting or exceeding $90 \%$ accuracy). The participants in the situational prevention focus condition were given an entry into the drawing for $\$ 50$ upon entry into the laboratory but were told that they had to exceed the same performance criterion in order to keep the entry. Importantly, none of the unidimensional rules would allow the participants to exceed the performance criterion, and thus discovery of the optimal rule required cognitive flexibility.

\section{Method}

\section{Participants}

One hundred eighteen participants from the university community completed the study and received either course credit or $\$ 6$ payment for participation in the experiment. Sixty participated in Experiment 1A, with 30 in each regulatory focus condition. Fiftyeight participated in Experiment 1B, with 28 in the situational promotion focus condition and 30 in the situational prevention focus condition. In addition, the participants were given the opportunity to obtain a 1 -in-10 chance of receiving $\$ 50$ in a random drawing (details provided below).

\section{Stimuli and Stimulus Generation}

The stimulus on each trial was a white line with some length and orientation presented against a black background vertically centered in the middle of a $650 \times 650$ pixel box, with some horizontal displacement from the left side of the box. A single set of 576 unique stimuli were samples, with 288 from Category A and 288 from Category B. This set was used for all the participants, but the order was randomized separately for each participant, with the constraint that the population parameters were preserved in each block. Category A consisted of stimuli sampled from 12 bivariate normal distributions on length and orientation, with 24 stimuli sampled from each. Category B consisted of stimuli sampled from 4 bivariate normal distributions on length and orientation, with 72 sampled from each. The value along the position dimension was generated independently, with the position values for Category A items being sampled from a univariate normal distribution with mean $=253$ pixels from the left side of the box and standard deviation $=75$ and the Category $\mathrm{B}$ items having mean $=397$ pixels and standard deviation $=75$. This yielded a wide range of positions and helped make the position di- 
mension especially salient. The category distribution parameters are outlined in Table 2 .

\section{Procedure}

The participants were tested individually in a dimly lit room. They were informed that there were two categories and that they should emphasize accuracy over speed of responding. In Experiment 1A, a correct response resulted in a gain of 2 points, and an incorrect response resulted in no gain or a loss of points. In Experiment 1B, a correct response resulted in a loss of 1 point, and an incorrect re-

Table 2

Category Distribution Parameters for the Experiments

\begin{tabular}{|c|c|c|c|c|c|}
\hline Category & $\mu_{1}$ & $\mu_{\mathrm{o}}$ & $\sigma_{1}$ & $\sigma_{\mathrm{o}}$ & $\operatorname{cov}_{10}$ \\
\hline \multicolumn{6}{|c|}{ Experiment 1} \\
\hline $\mathrm{A}_{1}$ & 42 & 42 & 12 & 12 & 0 \\
\hline $\mathrm{A}_{2}$ & 42 & 114 & 12 & 12 & 0 \\
\hline $\mathrm{A}_{3}$ & 42 & 186 & 12 & 12 & 0 \\
\hline $\mathrm{A}_{4}$ & 42 & 258 & 12 & 12 & 0 \\
\hline $\mathrm{A}_{5}$ & 114 & 42 & 12 & 12 & 0 \\
\hline $\mathrm{A}_{6}$ & 114 & 114 & 12 & 12 & 0 \\
\hline $\mathrm{A}_{7}$ & 114 & 186 & 12 & 12 & 0 \\
\hline $\mathrm{A}_{8}$ & 114 & 258 & 12 & 12 & 0 \\
\hline $\mathrm{A}_{9}^{\circ}$ & 186 & 42 & 12 & 12 & 0 \\
\hline $\mathrm{A}_{10}$ & 186 & 114 & 12 & 12 & 0 \\
\hline $\mathrm{A}_{11}$ & 258 & 42 & 12 & 12 & 0 \\
\hline $\mathrm{A}_{12}$ & 258 & 114 & 12 & 12 & 0 \\
\hline $\mathrm{B}_{1}$ & 186 & 186 & 12 & 12 & 0 \\
\hline $\mathrm{B}_{2}$ & 186 & 258 & 12 & 12 & 0 \\
\hline $\mathrm{B}_{3}$ & 258 & 186 & 12 & 12 & 0 \\
\hline $\mathrm{B}_{4}$ & 258 & 258 & 12 & 12 & 0 \\
\hline \multicolumn{6}{|c|}{ Experiment 2} \\
\hline $\mathrm{A}_{1}$ & 105 & 105 & 15 & 15 & 0 \\
\hline $\mathrm{A}_{2}$ & 105 & 135 & 15 & 15 & 0 \\
\hline $\mathrm{A}_{3}^{2}$ & 135 & 105 & 15 & 15 & 0 \\
\hline $\mathrm{A}_{4}$ & 135 & 135 & 15 & 15 & 0 \\
\hline $\mathrm{B}_{1}$ & 105 & 165 & 15 & 15 & 0 \\
\hline $\mathrm{B}_{2}$ & 105 & 195 & 15 & 15 & 0 \\
\hline $\mathrm{B}_{3}$ & 135 & 165 & 15 & 15 & 0 \\
\hline $\mathrm{B}_{4}$ & 135 & 195 & 15 & 15 & 0 \\
\hline $\mathrm{C}_{1}$ & 165 & 105 & 15 & 15 & 0 \\
\hline $\mathrm{C}_{2}$ & 165 & 135 & 15 & 15 & 0 \\
\hline $\mathrm{C}_{3}$ & 195 & 105 & 15 & 15 & 0 \\
\hline $\mathrm{C}_{4}$ & 195 & 135 & 15 & 15 & 0 \\
\hline $\mathrm{D}_{1}$ & 165 & 165 & 15 & 15 & 0 \\
\hline $\mathrm{D}_{2}$ & 165 & 195 & 15 & 15 & 0 \\
\hline $\mathrm{D}_{3}$ & 195 & 165 & 15 & 15 & 0 \\
\hline $\mathrm{D}_{4}$ & 195 & 195 & 15 & 15 & 0 \\
\hline \multicolumn{6}{|c|}{ Experiment 3} \\
\hline $\mathrm{A}_{1}$ & 40 & 110 & 12 & 12 & 0 \\
\hline $\mathrm{A}_{2}$ & 90 & 160 & 12 & 12 & 0 \\
\hline $\mathrm{A}_{3}$ & 140 & 210 & 12 & 12 & 0 \\
\hline $\mathrm{A}_{4}$ & 190 & 260 & 12 & 12 & 0 \\
\hline $\mathrm{A}_{5}$ & 240 & 310 & 12 & 12 & 0 \\
\hline $\mathrm{A}_{6}$ & 290 & 360 & 12 & 12 & 0 \\
\hline $\mathrm{B}_{1}$ & 110 & 40 & 12 & 12 & 0 \\
\hline $\mathrm{B}_{2}$ & 160 & 90 & 12 & 12 & 0 \\
\hline $\mathrm{B}_{3}$ & 210 & 140 & 12 & 12 & 0 \\
\hline $\mathrm{B}_{4}$ & 260 & 190 & 12 & 12 & 0 \\
\hline $\mathrm{B}_{5}$ & 310 & 240 & 12 & 12 & 0 \\
\hline $\mathrm{B}_{6}$ & 360 & 290 & 12 & 12 & 0 \\
\hline
\end{tabular}

Note-The orientation units were transformed into radians by multiplying each value by $\pi / 500$. For Experiments 1 and 3, the horizontal position for Category A items were sampled from a univariate normal distribution with mean $=253$ and standard deviation $=75$, whereas the Category B items were sampled from a univariate normal distribution with mean $=397$ and standard deviation $=75$. sponse resulted in a loss of 3 points. In Experiment 1A, the participants in the promotion focus condition were told that they would be given the opportunity to obtain an entry into a drawing for $\$ 50$ if they gained 86 or more points over the final 48 trials (the last block) of the experiment $(86=43$ of 48 trials correct $=90 \%$ correct $)$, whereas the participants in the prevention focus condition were given an entry into a drawing for $\$ 50$ upon arrival in the laboratory but were informed that they would lose the entry if they failed to gain 86 or more points over the final 48 trials of the experiment. In Experiment 1B, the participants in the promotion focus condition were told that they would be given the opportunity to obtain an entry into a drawing for $\$ 50$ if they lost 58 or fewer points over the final 48 trials (the last block) of the experiment $(-58=43$ of 48 trials correct $=90 \%$ correct), whereas the participants in the prevention focus condition were given an entry into a drawing for $\$ 50$ upon arrival in the laboratory but were informed that they would lose the entry if they failed to lose 58 or fewer points over the final 48 trials of the experiment. Both groups in both experiments were told that 10 participants were involved in the study and, thus, that they had at least a 1-in-10 chance of winning the drawing if they gained (or kept) their entry.

Each experimental session consisted of the presentation of twelve 48-trial blocks. At the beginning of each block, a vertically oriented point meter was set to zero. A sample screen for the gains reward structure used in Experiment $1 \mathrm{~A}$ is displayed in Figure 2A, and a sample screen for the losses reward structure used in Experiment $1 \mathrm{~B}$ is displayed in Figure 2B. The point meter was displayed on the right side of the computer screen and consisted of a 750 pixel tall $\times$ 50 pixel wide partially filled rectangle. The criterion was shown as a line across the meter and was labeled "Bonus." The region above the bonus line was labeled "Yes," and the region below "No," to indicate that the bonus was earned when the point meter was above the line and was not earned below the line. On each trial, a stimulus was displayed until the participant pressed either the A or the B key. Following the response, visual feedback was provided below the stimulus presentation box for $300 \mathrm{msec}$. If the response was correct, the word "Correct" was displayed. If the response was incorrect, the word "No, the correct category was X" was displayed, where X was the correct category for the stimulus. While this visual feedback remained on the screen, the point meter was updated. If the response was correct, the point meter filled from the bottom by 10 pixels (to denote 2 points) in the gains condition or emptied from the top by 5 pixels (to denote 1 point) in the losses condition. The region of change in the meter flashed three times ( $200 \mathrm{msec}$ for each flash) to emphasize that the meter was changing. In addition, the participant heard a cash register sound associated with each flash. There was a $100-\mathrm{msec}$ pause, and then the current point total was shown both graphically on the meter and in the text next to the point meter for $300 \mathrm{msec}$. If the response was incorrect, the point meter remained unchanged in the gains condition or emptied from the top by 15 pixels (to denote 3 points) in the losses condition, and the participant heard a buzzer for $600 \mathrm{msec}$, followed by a $100-\mathrm{msec}$ pause. For correct and incorrect responses, the stimulus display disappeared, but the point meter remained visible. There was a $250-\mathrm{msec}$ intertrial interval, followed by the initiation of the next trial.

At the end of each block of trials, the participants were given feedback on their performance in that block. If they remained above the bonus criterion in the promotion condition, they were told, "If that had been the last block of trials you would have earned an entry into the drawing for $\$ 50$." If they remained above the bonus criterion in the prevention condition, they were told, "If that had been the last block of trials you would have kept your entry into the drawing for $\$ 50$." If it was the final block, they were told whether they had gained or kept their entry into the drawing.

\section{Results and Theoretical Analyses}

We took a two-pronged approach to the data analysis. We began with an examination of the accuracy rates to 
determine how regulatory focus affected the accuracy of the participants' responding. Next, we applied a series of quantitative models to the data in an attempt to identify the types of strategies that the participants had used to solve the task and to determine what cognitive processes had been affected by the regulatory focus manipulation.

\section{Accuracy Analyses}

We conducted a number of separate analyses on the accuracy data to provide converging evidence in support of our hypothesis that a regulatory fit would lead to greater cognitive flexibility and, thus, to faster learning. We will present the analyses separately for Experiments 1A and $1 \mathrm{~B}$.

Experiment 1A. First, for each participant, we identified the first block of trials for which his or her accuracy met or exceeded the $90 \%$ performance criterion. This denotes the first block of trials for which the participant would have received feedback that, had that been the final block of trials, he or she would have earned an entry into the drawing (promotion focus) or would have kept the entry into the drawing (prevention focus). Any participant who failed to meet or exceed the criterion at any time during the experiment was coded with a 13 , because that would be the minimum number of blocks that a participant would need to achieve the criterion if he or she had not done it by the final (12th) block of trials. The results of a $t$ test confirmed that the participants given a promotion focus exceeded the performance criterion sooner (after 4.30 blocks, on average) than did the participants given a prevention focus (after 5.97 blocks, on average) $[t(58)=$ $1.70, p=.06]$.

Second, for each block of trials, we compared the proportion of promotion and prevention focus participants who met or exceeded the $90 \%$ performance criterion (see Figure 3A). We used a binomial test to determine whether the proportion of promotion participants who exceeded the criterion was significantly larger than the proportion of prevention participants who exceeded the criterion. The binomial test for the final block was significant $(p<.01)$, with $63.3 \%$ of the promotion participants, but only $36.7 \%$ of the prevention participants, meeting or exceeding criterion. Binomial tests conducted on a block-by-block basis suggested that the promotion advantage was significant in Blocks 3, 5, 10, and $11(p<.05)$. The differences were nonsignificant in every other block except Block 2 , which showed an advantage for the prevention regulatory focus. ${ }^{2}$ Collapsing across all blocks, the promotion focus participants exceeded criterion $40 \%$ of the time, whereas the prevention focus participants exceeded criterion only $27 \%$ of the time.

Finally, as is shown in Figure 3B, we computed the average accuracy in each block for the promotion and prevention participants. As was expected, the promotion participants were significantly more accurate $(91.3 \%)$ than the prevention participants $(86.5 \%)$ during the final block of trials $[t(58)=2.02, p<.05]$. A performance advantage for the promotion focus condition held in all blocks (except Block 4) and was significant, on the basis of a $t$ test, in Blocks $5[t(58)=2.53, p<.05]$ and $10[t(58)=$ 2.27, $p<.05]$ and marginally significant in Block 11 $[t(58)=1.75, p<.10]$. Thus, across all three measures, the promotion focus participants showed better learning, in support of the regulatory fit hypothesis.

Experiment 1B. The measures examined in Experiment $1 \mathrm{~A}$ were also examined in 1B. First, for each participant, we identified the first block of trials for which their accuracy met or exceeded the $90 \%$ performance criterion. In support of the regulatory fit hypothesis, the prevention focus participants exceeded the performance criterion sooner (after 3.43 blocks, on average) than did the promotion focus participants (after 4.25 blocks, on average), although this difference was nonsignificant $(t<1)$. These values are similar to those observed in Experiment 1A, although, under a losses reward structure, the performance criterion appears to be exceeded earlier, on average. Second, for each block of trials, we compared the proportion of promotion and prevention focus participants who met or exceeded the $90 \%$ performance criterion (see Figure 3D). Binomial tests were used to determine whether the proportion of prevention participants who exceeded the criterion was significantly larger than the proportion of promotion participants who exceeded the criterion. There was a prevention advantage in every block except Blocks 2 and 11, and the advantage was statistically significant in Blocks 3 , 4,6 , and $8(p<.05)$. Collapsing across all blocks, the prevention focus participants exceeded criterion $46 \%$ of the time, whereas the promotion focus participants exceeded criterion only $34 \%$ of the time. Again, these findings are in line with the regulatory fit hypothesis and are quite similar to the values observed in Experiment 1A, in which $40 \%$ of the regulatory fit participants (i.e., those with a promotion focus), on average, exceeded criterion, whereas only $27 \%$ of the regulatory mismatch participants (i.e., those with a prevention focus) exceeded the criterion. Finally, we computed average accuracy in each block for the promotion and prevention participants (see Figure 3E). Collapsed across blocks, accuracy rates were higher for the prevention participants $(87.2 \%)$ than for the promotion participants $(84.4 \%)[t(56)=2.08, p<.05]$. In addition, a performance advantage was observed for the prevention participants in all the blocks except Blocks 9 and 11. This difference was statistically significant in Blocks 3 $[t(56)=2.91, p<.01], 6[t(56)=2.76, p<.01]$, and 8 $[t(56)=1.97, p<.05]$ and was marginally significant in Block $4[t(56)=1.91, p=.06]$. These average accuracy rates are consistent with the regulatory fit hypothesis and are similar to the values observed in Experiment 1A, in which the regulatory fit participants (those with a promotion focus) achieved $86.8 \%$ average accuracy, whereas the regulatory mismatch participants (those with a prevention focus) achieved $84.5 \%$ average accuracy.

Taken together, the accuracy-based analyses from Experiments $1 \mathrm{~A}$ and $1 \mathrm{~B}$ converge in suggesting that participants in a regulatory fit (i.e., a situational promotion focus with gains or a situational prevention focus with losses) (1) exceed the performance criterion earlier in training, (2) are more likely, in general, to exceed the performance 


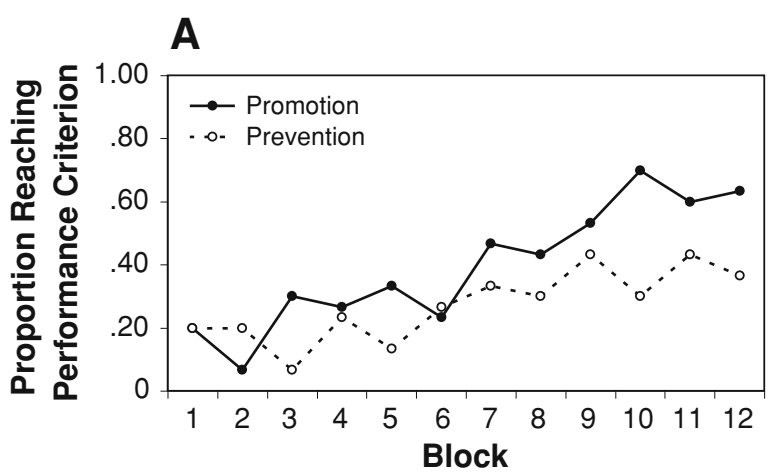

B
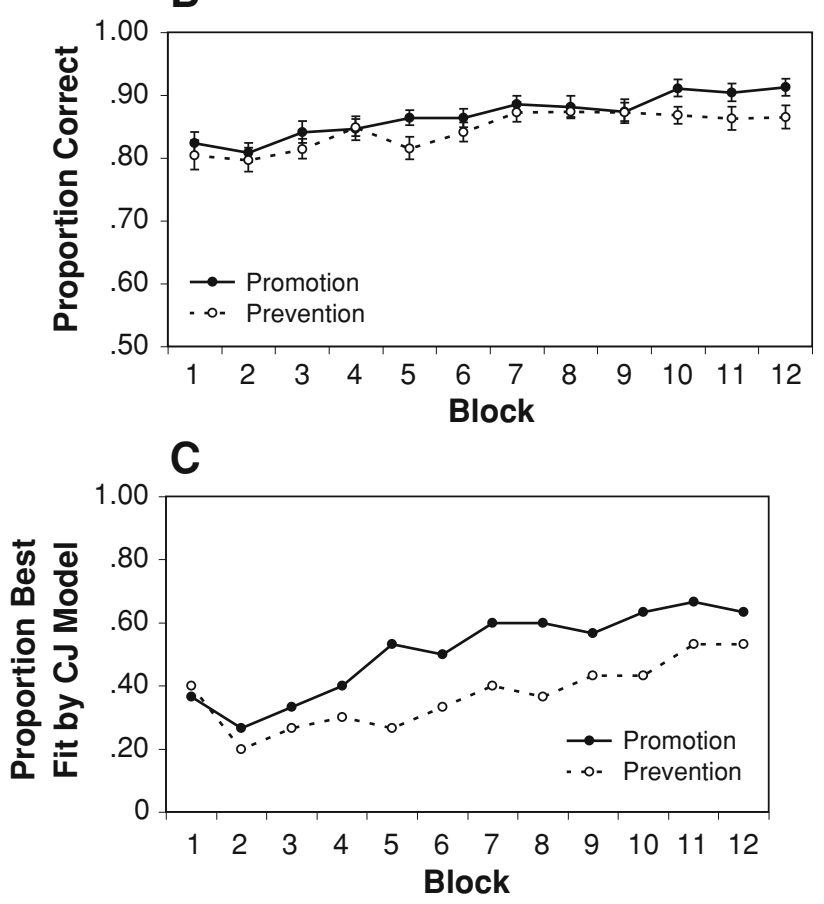

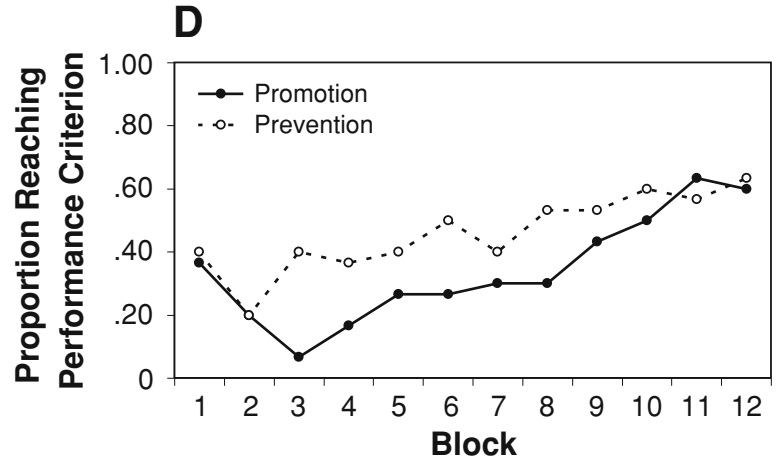

$\mathbf{E}$
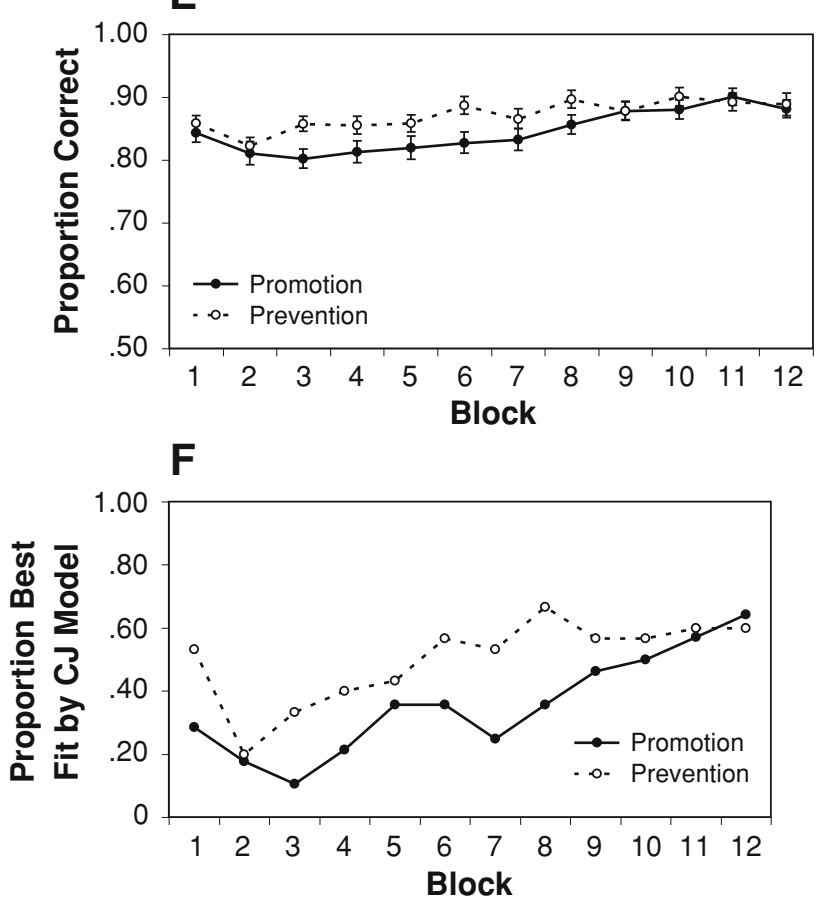

Figure 3. (A) Proportion of participants who exceeded the performance criterion in Experiment 1A (gains). (B) Proportion correct (averaged across observers) in Experiment 1A, along with standard error bars. (C) Proportion of participants whose data were best fit by a conjunctive model in Experiment 1A. (D) Proportion of participants who exceeded the performance criterion in Experiment 1B (losses). (E) Proportion correct (averaged across observers) from Experiment 1B, along with standard error bars. (F) Proportion of participants whose data were best fit by a conjunctive model in Experiment 1B.

criterion necessary to earn (or keep) an entry into a drawing, and (3) obtain higher overall accuracy rates than do participants in a regulatory mismatch (i.e., a situational prevention focus with gains or a situational promotion focus with losses).

\section{Model-Based Analyses}

The accuracy-based analyses support the proposal that a regulatory fit increases cognitive flexibility, leading participants to learn more quickly and attain a higher accuracy rate. To determine whether the improved performance was due to regulatory fit participants' (the situational promotion focus participants in Experiment $1 \mathrm{~A}$ and the situational prevention focus participants in Experiment 1B) abandoning simple unidimensional rules in favor of the more complex, optimal conjunctive rule more quickly than did the regulatory mismatch participants (the situational prevention participants in Experiment $1 \mathrm{~A}$ and the situational promotion participants in Experiment 1B), we fit five decision bound models (Ashby \& Maddox, 1993; Maddox \& Ashby, 1993) separately to the data from each participant on a block-by-block basis. ${ }^{3}$ All the analyses were performed at the individual-participant level, because of concerns with modeling aggregate data (e.g., Ashby, Maddox, \& Lee, 1994; Estes, 1956; Maddox, 1999; Maddox \& Estes, 2004; Smith \& Minda, 1998). The optimal conjunctive rule model assumes that the participant uses the optimal criterion along length and orientation. This model has one free parameter that represents the variance of internal (perceptual and criterial) noise. The suboptimal 
conjunctive rule model allows the length and orientation decision criteria to be estimated from the data and has three parameters (two for the decision criteria and one for the variance of internal noise). The unidimensional length model assumes that the participant sets a criterion on length and gives one response for short lines and the other response for long lines and completely ignores orientation and position. The unidimensional orientation model assumes that the participant sets a criterion on orientation and gives one response for shallow lines and the other response for steep lines and completely ignores length and position. The unidimensional position model assumes that the participant sets a criterion on position and gives one response for lines to the left and the other response for lines to the right and completely ignores length and orientation. Each of the three unidimensional models contains two free parameters (i.e., one decision criterion and the noise variance).

The model parameters were estimated using maximum likelihood (Ashby, 1992; Wickens, 1982), and the goodnessof-fit statistic was

$$
\mathrm{AIC}=2 r-2 \cdot \ln L,
$$

where $r$ is the number of free parameters and $L$ is the likelihood of the model, given the data (Akaike, 1974; Takane \& Shibayama, 1992). The smaller the AIC, the closer a model is to the "true model," regardless of the number of free parameters. Thus, the best model is the one with the smallest AIC value (for a discussion of the complexities of model comparisons, see Myung, 2000; Pitt, Myung, \& Zhang, 2002).

Experiment 1A. Table 3 displays the percentage of the promotion and prevention participants' data best fit by a conjunctive rule model, the unidimensional position model, and the unidimensional length or unidimensional orientation model separately by block. ${ }^{4}$ For ease of comparison with the accuracy data, we also plot the proportion of promotion and prevention participants' data best fit by a conjunctive rule in Figure 3C. In addition, we present the average accuracy rate for the participants in each model class. Before summarizing the model results, it is important to determine whether the models provided a good account of the data. Poor-fitting models should not be interpreted. The models fit the data well, with the most general, suboptimal conjunctive rule model accounting for $92 \%$ and $86 \%$ of the final block responses for the promotion and the prevention condition participants, respectively.

Following the approach taken with the accuracy data, we conducted a number of separate analyses on the modeling results, in an attempt to provide converging evidence in support of the hypothesis that participants in a regulatory fit (i.e., those with a promotion focus) have greater cognitive flexibility and, thus, show faster learning. First, as was expected, the unidimensional length and unidimensional orientation models rarely fit the data best, providing the best fit for over $25 \%$ of the data in only the first block of trials for the promotion and prevention participants and rarely fitting best for more than $10 \%$ of the data in any other block. In contrast, the proportion of data sets best fit by the unidimensional position model was high in all but Blocks 1 and 3 and was higher for the prevention participants than for the promotion participants. This pattern suggests that the prevention focus participants were less likely to abandon this simple rule in favor of the more complex conjunctive rule. Second, we identified the first block of trials for which a conjunctive rule model provided the best account of the data separately for each participant. Any participant whose data were never best fit by a conjunctive model was coded with a 13 . The results of a $t$ test confirmed that the promotion participants' data were best fit by a conjunctive model earlier (after 3.87 blocks, on average), in comparison with the prevention participants' data (after 6.07 blocks, on average) $[t(58)=1.830$, $p<.05]$. Finally, we determined whether the proportion of participants whose data were best fit by a conjunctive

Table 3

Percentages of Promotion and Prevention Focus Participants for Which a Conjunctive, Unidimensional Position, Unidimensional Length, or Unidimensional Orientation Model Provided the Best Account of the Data, Along With the Average Accuracy Rates (Proportion Correct $[P]$ ) Obtained for Those Participants in Experiment 1A

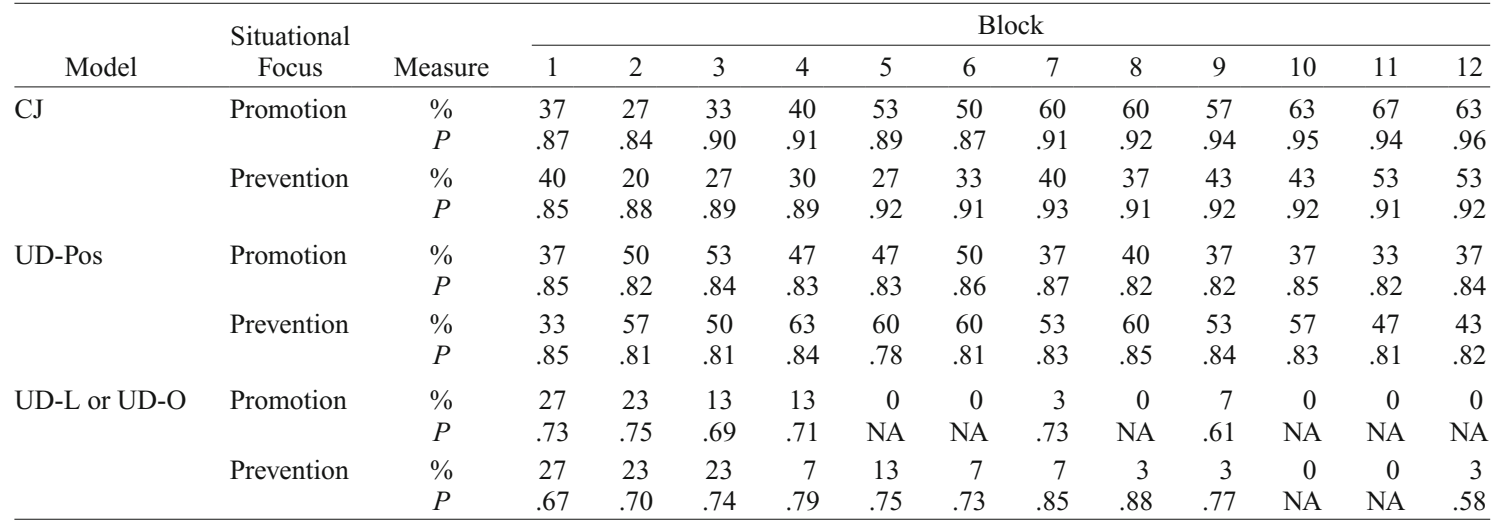

Note - CJ, conjunctive rule model; UD-Pos, unidimensional position model; UD-L, unidimensional length model; UD-O, unidimensional orientation model. 
rule model was higher for the promotion participants than for the prevention participants, using binomial tests. Although the proportion of promotion participants whose data were best fit by a conjunctive rule was larger than that for the prevention participants in all but the first block of trials, the promotion focus advantage was statistically significant only in Blocks 5, 6, 7, 8, and $10(p<.05) .^{5}$

Taken together, these results suggest that when there is a gains reward structure, promotion focus participants are more cognitively flexible than prevention participants, because they abandon the simple unidimensional rule in favor of the more complex conjunctive rule more quickly. We expected that after a participant had abandoned the unidimensional rule for a conjunctive rule, learning should proceed at approximately the same rate regardless of regulatory fit. To test this hypothesis, we examined the change in the noise parameter across blocks for the promotion and the prevention participants' best fits by a conjunctive rule model. Specifically, we identified the first block of trials for which a conjunctive rule model fit each participant's data and then tracked the noise value from that "first" block four additional blocks out. We analyzed only five blocks from the first use of a conjunctive rule, because many of the participants completed the experiment within five blocks of using the conjunctive rule for the first time. The average noise standard deviations were 44, 36, 26, 34, and 28 for the promotion condition and $41,35,31,34$, and 29 for the prevention condition. All the $t$ tests comparing the promotion and prevention noise values on a blockby-block basis were nonsignificant (all $t \mathrm{~s}<1$ ), supporting our claim that promotion and prevention participants' performance profiles should be the same once they shift to a conjunctive model. An examination of the best-fitting decision criteria revealed an identical pattern.

Experiment 1B. Table 4 displays the percentage of promotion and prevention participants' data best fit by a conjunctive rule model, the unidimensional position model, and either the unidimensional length or the uni- dimensional orientation model separately by block, along with the average accuracy rate for the participants in each model class. Figure 3F plots the proportion of promotion and prevention participants' data best fit by a conjunctive rule. As in Experiment 1A, the best-fitting model from the final block of trials accounted for $92 \%$ and $90 \%$ of the responses for the prevention and the promotion condition participants, respectively, suggesting a good account of the data.

As was expected, and in line with the results from Experiment $1 \mathrm{~A}$, the unidimensional length and unidimensional orientation models rarely fit the data best, providing the best fit for over $25 \%$ of the data only in the first block of trials for the promotion participants and fitting best only $8 \%$ of the time overall. On the other hand, the proportion of data sets best fit by the unidimensional position model was high ( $44 \%$ for the prevention participants and $62 \%$ for the promotion participants, on average) and was higher in all the blocks for the promotion participants than in those for the prevention participants. Also as was predicted from the regulatory fit hypothesis, the prevention participants' data were best fit by a conjunctive model earlier (after 3.5 blocks, on average), in comparison with the promotion participants (after 5.2 blocks, on average). This difference was marginally significant $[t(56)=1.55$, $p=.06]$. Finally, the proportion of participants whose data were best fit by a conjunctive rule model was higher for the prevention participants than for the promotion participants. An examination of Figure $3 \mathrm{~F}$ suggests that more prevention participants than promotion participants shifted away from unidimensional strategies to conjunctive strategies in all but the final block. Binomial tests suggested that the prevention advantage was significant in Blocks $1,3,4,6,7$, and $8(p<.05)$.

Following the approach taken in Experiment 1A, we examined the change in the noise parameter across blocks for the promotion and prevention participants best fit by a conjunctive rule model. We identified the first block of

Table 4

Percentages of Promotion and Prevention Focus Participants for Which a Conjunctive, Unidimensional Position, Unidimensional Length, or Unidimensional Orientation Model Provided the Best Account of the Data, Along With the Average Accuracy Rates (Proportion Correct $[P]$ ) Obtained for Those Participants in Experiment 1B

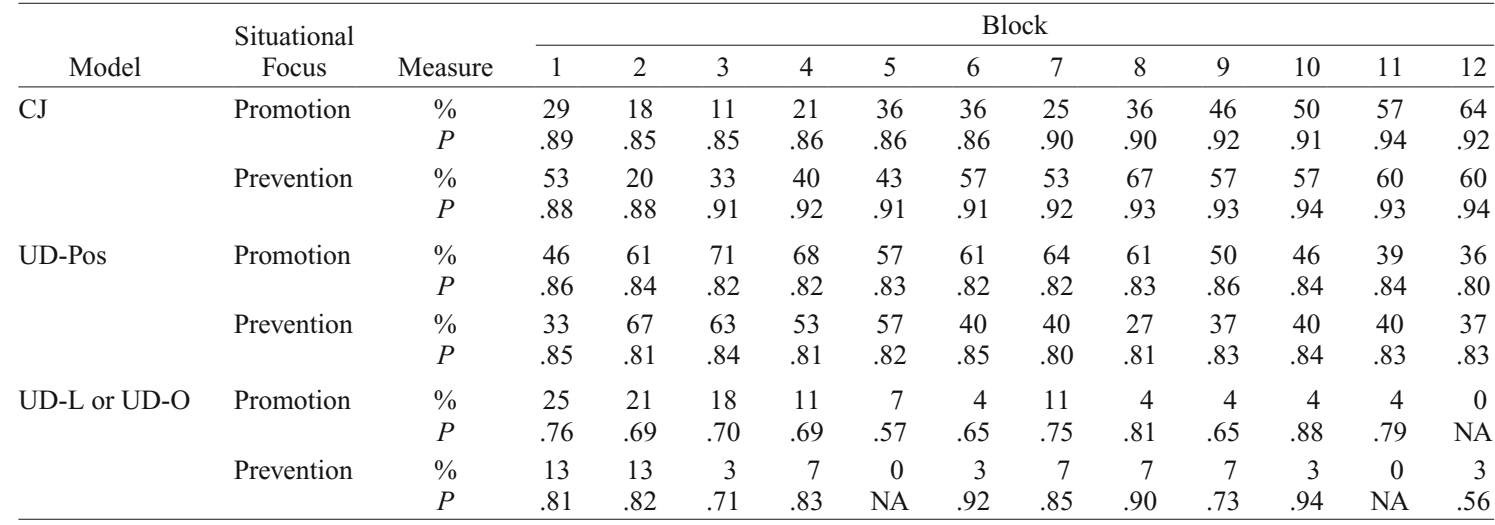

Note-CJ, conjunctive models (includes CJ-suboptimal and CJ-optimal); UD-Pos, unidimensional position model; UD-L or UD-O, unidimensional length or orientation model. 
trials for which a conjunctive rule model fit each participant's data and tracked the noise value four additional blocks out. The average noise standard deviations were 42 , $50,54,40$, and 35 for the promotion condition and 40, 47, 44,43 , and 43 for the prevention condition. Although not monotonic, there was a general trend toward decreasing noise values. Most important, there were no significant differences in the noise values across the promotion and prevention participants in any block (largest $t$ value $=$ 1.16), suggesting that promotion and prevention participants' performance profiles should be the same once they shift to a conjunctive model. Analyses of the decision criterion yielded a similar result.

\section{Discussion}

In Experiment 1, we tested the hypothesis that a regulatory fit between the situational regulatory focus and the reward structure of the task would lead to greater cognitive flexibility, using a rule-based perceptual classification task for which flexibility was advantageous. In Experiment 1A, we used a gains reward structure, and in Experiment 1B, we used a losses reward structure. The regulatory fit hypothesis predicts that situational promotion focus participants should perform better in Experiment $1 \mathrm{~A}$, whereas situational prevention focus participants should perform better in Experiment 1B. As was predicted, the participants in a regulatory fit exceeded the performance criterion sooner and achieved a higher overall level of performance during the final blocks of trials than did the people in a regulatory mismatch. The model-based analyses suggested that the participants in a regulatory fit shifted away from a unidimensional rule strategy toward a conjunctive rule strategy sooner than did the people in a regulatory mismatch, resulting in quicker, better learning. Interestingly, though, once a regulatory fit or regulatory mismatch participant abandoned the unidimensional rule in favor of the conjunctive rule, their learning profile was the same. The latter finding suggests that the regulatory fit manipulation affected the speed of the shift from unidimensional to conjunctive rule use but not the adjustments to the conjunctive rule decision criteria, once that shift had been achieved.

On the basis of the results of Experiment 1 only, one might draw the conclusion that a regulatory fit leads to better performance in classification tasks than does a regulatory mismatch. This fit advantage might hold regardless of whether cognitive flexibility is advantageous or not. In Experiment 2, we tested this hypothesis with a gains reward structure, and in Experiment 3, we tested this hypothesis with a losses reward structure.

\section{EXPERIMENT 2}

To determine whether a regulatory fit leads to better classification learning, regardless of the need for flexibility cognitive processing, or whether a regulatory fit leads to increased cognitive flexibility and, thus, to poor performance when cognitive flexibility is disadvantageous, we designed a rule-based classification task for which conservative changes in a person's criterion would lead to better performance in the task than would large changes that might be associated with high flexibility. As in Experiment 1 , we used a conjunctive-rule-based classificationlearning task, but instead of a two-category problem for which unidimensional rules existed that yielded good performance, we used a four-category problem for which it was clear early in learning that a two-dimensional rule was required (see Figure 1B). The stimulus on each trial was a line whose length and orientation varied across trials. The optimal rule can be described as follows: "Respond A if the length is short and the orientation is shallow, respond $\mathrm{B}$ if the length is short and the orientation is steep, respond $\mathrm{C}$ if the length is long and the orientation is shallow, and respond D if the length is long and the orientation is steep." Because the two-dimensional nature of the optimal rule is clear early in learning, the type of cognitive flexibility associated with qualitative shifts in the nature of the decision rule will not be useful. Instead, good performance will be achieved through slower, more incremental changes in the decision criterion values. These slower, more incremental changes should be associated with lower levels of cognitive flexibility. In Experiment 2, we used a gains reward structure and, thus, predicted that regulatory mismatch participants (i.e., those with a situation prevention focus) should perform better.

Two additional characteristics of the task were added to bias the task in favor of low cognitive flexibility. First, the category distributions overlapped, requiring the participants to learn the noisy criterion between short and long lines and between shallow and steep orientations. Second, a base rate manipulation was introduced. Specifically, Categories C and D were presented three times as often as Categories A and B. Base rate manipulations affect the location of the optimal decision criterion and, in the present experiment, shifted the location of the optimal length criterion away from the equal likelihood criterion. When the base rates are unequal, the optimal classifier sacrifices accuracy on the low base rate categories to increase accuracy on the more prevalent high base rate categories, effectively increasing overall accuracy and long-run reward. In the present study, optimal accuracy was $77 \%$ for Categories A and $\mathrm{B}$ and $89 \%$ for Categories $\mathrm{C}$ and $\mathrm{D}$. Thus, the fact that (1) the two-dimensional nature of the optimal rule is clear early, (2) the locations of the optimal criteria are noisy due to category overlap, and (3) the base rate difference shifts the criteria away from the equal likelihood criteria should provide an environment that fits well with the low cognitive flexibility, prevention focus manipulation.

The regulatory focus manipulation is one that is fairly subtle and, thus, should not be expected to lead to differential patterns of performance under all conditions. For example, if one constructs a situation in which the bonus is easily attainable even with a suboptimal rule, there should be little effect of regulatory focus, because both promotion and prevention focus participants should achieve the goal easily. In contrast, if we create a situation in which the bonus is rarely or never attainable, we should observe larger effects of the situational regulatory focus 
manipulation. To investigate this possibility, we examined the effects of situational regulatory focus (promotion vs. prevention) on four-category conjunctive-rule-based classification learning under two goal states, one for which the bonus was easily attainable (the attainable goal condition) and one for which the bonus was unattainable (the unattainable goal condition). This resulted in a 2 (situational regulatory focus) $\times 2$ (goal state) factorial design.

Situational focus was manipulated using the same approach as that in Experiment 1. The participants in the attainable goal condition received 2 points for each correct response and no points for incorrect responses. The participants in the attainable goal condition could obtain or keep the drawing entry if they achieved $75 \%$ of the performance level obtained by the optimal classifier. The participants in the unattainable goal condition received 1 point for each correct response and no points for incorrect responses. The participants in this condition could not obtain or keep the drawing entry even if they responded correctly on every trial in the experiment.

We predicted that situational regulatory focus and goal state should interact in their effect on performance. Specifically, we predicted no effect of situational focus when the goal state was highly attainable because both the promotion and the prevention participants could easily achieve the stated goal. In contrast, we predicted a strong effect of situational focus on performance when the goal state was unattainable. Under these conditions, the promotion and prevention participants were unable to achieve the goal state but were attempting to do so throughout the session. If a promotion focus leads to high levels of cognitive flexibility, the promotion focus participants should be biased toward large changes in their decision criteria across trials. In contrast, if a prevention focus leads to lower levels of cognitive flexibility, the prevention focus participants should be biased toward smaller changes in their decision criteria across trials. Learning of noisy decision criteria under unequal base rate conditions should be better when small decision criterion changes are made. Thus, this task was better suited for a situational prevention focus.

\section{Method}

\section{Participants}

One hundred three participants from the university community completed the study and received course credit or $\$ 6$ payment for participation. In addition, the participants were given the opportunity to obtain a 1 -in-10 chance of receiving $\$ 50$ in a random drawing. Twenty-five participated in the promotion-attainable condition, with 26 participating in the promotion-unattainable, preventionattainable, and prevention-unattainable conditions. No one participated in more than one condition.

\section{Stimuli and Stimulus Generation}

The stimulus on each trial was a white line with some length and orientation presented against a black background centered in the middle of a $650 \times 650$ pixel box in the upper left corner of the computer screen. Because the categories overlapped, optimal accuracy was $77 \%$ for the low base rate categories and $89 \%$ for the high base rate categories. Seventy-two unique stimuli were generated from the two low base rate categories (A and B), and 216 unique stimuli were generated from the two high base rate categories (C and D), for a total of 576 unique stimuli. Each category consisted of stimuli from four bivariate normal distributions. Eighteen items were sampled from each of the A and B subcategories, whereas 54 were sampled from each of the $\mathrm{C}$ and $\mathrm{D}$ subcategories. The category distribution parameters are outlined in Table 2, and a scatterplot of the stimuli, along with the optimal bounds, is displayed in Figure 1B. The stimuli were randomized separately for each participant.

\section{Procedure}

The participants were tested individually in a dimly lit room. They were informed that there were four categories and that they should emphasize accuracy over speed of responding. There was no mention of the base rate manipulation. The participants in the promotion focus condition were told that they would be given the opportunity to obtain an entry into a drawing for $\$ 50$ if they gained 67 or more points over the final 48 trials (the last block) of the experiment. The participants in the prevention focus condition were given an entry into a drawing for $\$ 50$ upon arrival in the laboratory but were informed that they would lose the entry if they did not gain 67 or more points over the final 48 trials of the experiment. In the attainable goal condition, a correct response resulted in a gain of 2 points, whereas in the unattainable goal condition, a correct response resulted in a gain of only 1 point. In both conditions, no points were gained or lost for an incorrect response. In the attainable goal condition, the ideal observer obtains 90 points in a block of trials. On the basis of pilot research, we found that nearly all participants can reach $75 \%$ of optimal or 67 points within a few blocks (usually three or four). All other aspects of the experimental procedure were identical to those in Experiment 1.

\section{Results and Theoretical Analyses}

As in Experiment 1, we will begin with an examination of the accuracy rates, to determine how situational regulatory focus and goal state affected the accuracy of the participants' responses. Then we will turn to the models, in an attempt to identify what cognitive processes were affected by the situational focus and goal state manipulations.

\section{Accuracy Analyses}

Because the goal was highly attainable in the attainable goal state conditions, we expected that the promotion and prevention participants would meet or exceed the performance criterion early in learning and at the same rate. As a test of this prediction, we determined the first block of trials for which each promotion and prevention participant met or exceeded the goal. As was expected, both groups achieved the goal early (average for promotion $=2.76$; prevention $=3.35$ ), and there was no difference across regulatory focus groups $[t(49)=1.16$, $p>.05]$. The same analyses could not be conducted on the unattainable goal participants, since the goal was not attainable even if the participant was correct on every trial. Thus, to determine how situational regulatory focus and goal state affected performance, we conducted a 2 (regulatory focus: promotion vs. prevention) $\times 2$ (goal state: attainable vs. unattainable) $\times 12$ (block) ANOVA on the proportion correct data. Figure 4A displays the proportions correct (averaged across participants) for the twelve 48-trial blocks for the promotion-attainable, promotion-unattainable, prevention-attainable, and prevention-unattainable conditions. The main effects of regulatory focus $\left[F(1,99)=5.41, M S_{\mathrm{e}}=0.08, p<\right.$ 


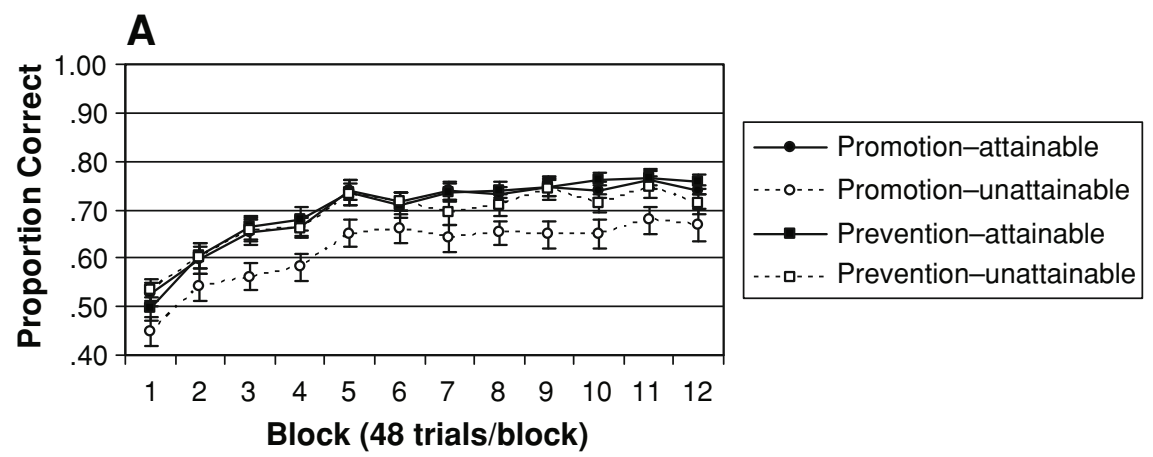

B

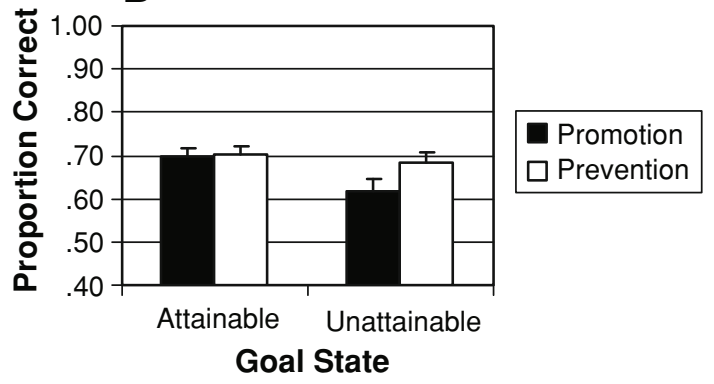

Figure 4. (A) Proportion correct (averaged across observers) in Experiment 2, along with standard error bars. (B) Proportion correct (averaged across participants and blocks) in Experiment 2, along with standard error bars.

$.05]$, goal state $\left[F(1,99)=9.30, M S_{\mathrm{e}}=0.08, p<.01\right]$, and block $\left[F(11,1089)=60.46, M S_{\mathrm{e}}=0.01, p<.001\right]$ were all significant. Block did not interact with regulatory focus, goal state, or the combination (all $F \mathbf{s}<1$ ), but the regulatory focus $\times$ goal state interaction was significant $\left[F(1,99)=4.38, M S_{\mathrm{e}}=0.08, p<.05\right]$. The interaction is depicted in Figure 4B. Three comments are in order. First, regulatory focus had no effect on performance when the goal was attainable $(t<1)$. Second, performance in the prevention-unattainable-goal condition was equivalent to performance for the promotion- and preventionattainable-goal conditions $(t \mathrm{~s}<1)$. Finally, performance in the promotion-unattainable-goal condition was significantly worse than that in each of the other three conditions $(p \mathrm{~s}<.01)$. Taken together, these data suggest that a situational prevention focus (i.e., a regulatory mismatch) leads to better conjunctive-rule-based classification learning when the goal is unattainable and cognitive flexibility is disadvantageous. When the goal is attainable, regulatory focus has no effect on performance.

\section{Model-Based Analyses}

Four decision bound models were fit separately to each participant's block-by-block data. ${ }^{6}$ Each assumes that the participant used a conjunctive, rule-based strategy, but the models differ in their assumptions about the optimality of the decision criterion placement along the length and orientation dimensions. The optimal model assumes that the participant used the length and orientation criteria that maximized performance. This model has only internal noise variance as a free parameter. The suboptimal length model allows the length criterion to be estimated from the data, whereas the suboptimal orientation model allows the orientation criterion to be estimated from the data. These two models contain two free parameters (i.e., one criterion and the noise variance). The suboptimal length-orientation model allows the length and orientation criteria to be estimated from the data (along with the noise variance).

AIC was used to determine the best-fitting model for each participant separately by block. As one might expect, performance became more nearly optimal over blocks. Interestingly, there was a shift from the suboptimal lengthorientation model's dominating early toward better fits of the suboptimal length model. Recall that the latter model assumes the optimal decision criterion along the orientation dimension and a suboptimal decision criterion along the length dimension. This pattern was expected, since the base rate manipulation was along the length dimension and it is well established that participants underestimate the criterion shift associated with optimal responding when base rates are unequal (see Maddox, 2002, for a review). Despite this one interesting aspect of the modelbased-learning profile, the most important model-based results can be obtained by examining model performance averaged across blocks.

Table 5 displays the percentage of participants whose data were best fit by each of the models (averaged across the 12 blocks), along with the average accuracy rate for each class of participants separately for the four situational regulatory focus $\times$ goal state conditions. Two main comments are in order. First, the percentage of participants 
Table 5

Percentages of Participants (Averaged Across Blocks) for Which Each of the Four Models Provided the Best Account of the Data, Along With the Average Accuracy Rates Obtained for Those Participants in Experiment 2

\begin{tabular}{|c|c|c|c|c|c|}
\hline \multirow[b]{3}{*}{ Model } & \multirow[b]{3}{*}{ Measure } & \multicolumn{4}{|c|}{ Condition } \\
\hline & & \multicolumn{2}{|c|}{ Attainable } & \multicolumn{2}{|c|}{ Unattainable } \\
\hline & & Promotion & Prevention & Promotion & Prevention \\
\hline \multirow[t]{2}{*}{ Optimal } & Model percentage & 22 & 26 & 23 & 27 \\
\hline & Proportion correct & .69 & .68 & .60 & .70 \\
\hline \multirow[t]{2}{*}{ Suboptimal orientation } & Model percentage & 16 & 12 & 15 & 13 \\
\hline & Proportion correct & .71 & .73 & .62 & .69 \\
\hline \multirow[t]{2}{*}{ Suboptimal length } & Model percentage & 43 & 38 & 36 & 38 \\
\hline & Proportion correct & .70 & .69 & .63 & .67 \\
\hline \multirow[t]{2}{*}{ Suboptimal, both } & Model percentage & 19 & 25 & 26 & 22 \\
\hline & Proportion correct & .69 & .71 & .61 & .70 \\
\hline
\end{tabular}

best fit by each model was relatively unaffected by the regulatory focus and goal state manipulations, although a slightly higher percentage of participants used the optimal decision strategy in the prevention-unattainable condition. Second, of the four models, the suboptimal length model provided the best fit for approximately $40 \%$ of the participants across conditions. This finding, along with the fact that approximately $20 \%$ of the participants' data were best fit by the optimal model, suggests that approximately $60 \%$ of the participants in each condition were using the optimal decision criterion along orientation, but not along length.

As was outlined earlier, we predicted that a situational promotion focus should lead to greater cognitive flexibility in the form of larger shifts in the decision criteria across trials when the goal was unattainable. Large shifts in the decision criteria across trials would result in a larger estimate of the noise parameter in the model. Thus, we predicted larger noise estimates in the promotion-unattainable condition than in the other three conditions. In addition, we predicted no difference in the noise estimates across these three remaining conditions. As a test of this hypothesis, we examined the parameters of the suboptimal length-orientation model. This model is the most general model and thus, by definition, must provide the best account of the data. In fact, during the final block of trials, this model accounted, on average, for $82 \%, 84 \%, 73 \%$, and $80 \%$ of the participants' responses in the promotion-attainable, prevention-attainable, promotion-unattainable, and prevention-unattainable conditions, respectively. The estimated standard deviation of internal noise from the suboptimal length-orientation model was subjected to a 2 (regulatory focus) $\times 2$ (goal state) $\times 12$ (block) mixed design ANOVA. All three main effects were significant [regulatory focus, $F(1,99)=$ $5.20, M S_{\mathrm{e}}=2,836.34, p<.05$; goal state, $F(1,99)=$ $8.69, M S_{\mathrm{e}}=630.38, p<.01$; block, $F(11,1089)=86.31$, $\left.M S_{\mathrm{e}}=221.12, p<.001\right]$, but none of the interactions was significant. Although the regulatory focus $\times$ goal state interaction was only marginally significant $[F(1,99)=$ $\left.2.73, M S_{\mathrm{e}}=2,836.34, p=.10\right]$, we conducted follow-up analyses comparing the promotion-unattainable condition noise values with those from the other three conditions.
These data are depicted in Figure 5. As was expected, we found that the standard deviation of internal noise was statistically equivalent across the two attainable goal conditions and the prevention-unattainable goal condition but that there was significantly greater variability in the promotion-unattainable goal condition than in any other condition (all $p \mathrm{~s}<.05$ ). It is worth mentioning that an examination of the best-fitting decision criteria yielded a similar result, with larger deviations from the optimal values in the promotion-unattainable condition than in the other three conditions that yielded similar decision criterion values.

\section{Discussion}

In Experiment 2, we examined the effects of regulatory focus on four-category, conjunctive-rule-based classification learning when the goal was easily attainable or unattainable and a gains reward structure was present. Because the two-dimensional nature of the rule was clear early in learning, and because noisy decision criteria had to be learned under unequal base rate conditions, we expected that classification learning would be best when the participant was under a motivational state conducive to gradual, incremental adjustment of the decision criteria, as opposed to a motivational state conducive to large shifts in the decision criteria. Our working hypothesis was that

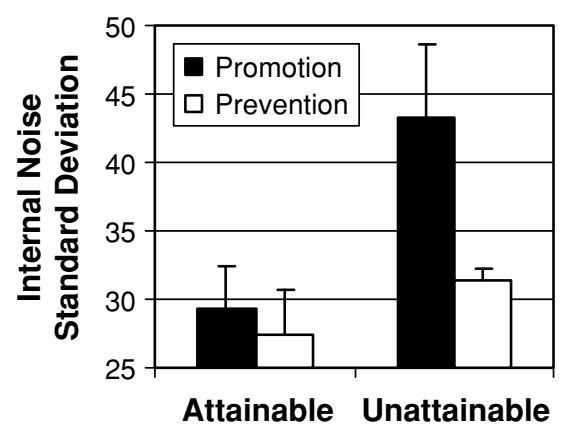

Figure 5. Average internal noise standard deviation for the suboptimal-both model (averaged across participants) in Experiment 2, along with standard error bars. 
a promotion focus results in a high level of cognitive flexibility because of the regulatory fit with the gains reward structure and, thus, is associated with large criterion shifts, whereas a prevention focus results in low levels of cognitive flexibility and, thus, is associated with more gradual shifts in criterion. We predicted that this regulatory focus effect would be observed only when the goal was difficult (or impossible) to attain.

The results supported our prediction. Performance in the attainable goal condition was unaffected by regulatory focus and was equivalent to performance in the unattainable goal condition when a prevention focus was induced. In contrast, performance was significantly worse in the unattainable goal condition when a promotion focus (regulatory fit) was induced. The model-based analyses suggest that the locus of this performance deficit in the latter condition is due to poorer decision criterion placement and greater variability in the memory and placement of the decision criterion. Both of these problems can be predicted from the cognitive flexibility hypothesis, because large changes in the decision criteria will lead to poorer learning of the optimal criteria and, by definition, will be more variable.

\section{EXPERIMENT 3}

In Experiment 2, we used a gains reward structure and a rule-based category structure for which the rule was obvious early in learning to test the hypothesis that a regulatory fit would be disadvantageous when cognitive flexibility was disadvantageous. To increase the likelihood that cognitive flexibility would be disadvantageous, we utilized overlapping categories and unequal base rates. Importantly, we took a first step toward examining the influence of goal state - in particular, its attainability. We found support for the regulatory fit hypothesis in that prevention focus participants (those in a regulatory mismatch) showed better learning, but only when the goal was unattainable.

In Experiment 3, we also used a category structure for which cognitive flexibility was disadvantageous but examined a losses reward structure. Rather than continue to examine the influence of goal state and use unequal base rate, overlapping categories, in Experiment 3 we decided to take a more direct approach and to extend our investigation to the use of information integration categories. In Experiment 3, we used procedures that were as similar as possible to those in Experiment 1, the category structure displayed in Figure 1C. Note that the stimulus dimensions were identical to those used in Experiment 1. Accuracy for the optimal information integration rule was $100 \%$, and the most accurate unidimensional rule on the highly salient position dimension yielded 83\%, as in Experiment 1 . The losses reward structure, bonus criterion, and manipulation of regulatory focus in Experiment 1 were used. The main (but critical) difference was that the optimal rule required information integration.

With information integration category structures such as those in Figure 1C, conjunctive rules can often be utilized that lead to higher levels of performance than do unidimensional rules. We were aware of this possibility when designing the task and were careful to construct an information integration rule for which the conjunctive rules yielded accuracy below the $90 \%$ criterion level. This is not a trivial task, but we were able achieve our aim. The most accurate conjunctive rule that utilized length and orientation yielded $78 \%$ correct. The most accurate conjunctive rules that utilized position and length or position and orientation yielded higher accuracy rates of $86 \%$.

COVIS assumes that a procedural-based learning system dominates information integration category learning. On the basis of the proposed link between frontal brain structures, cognitive flexibility, and processing in the hypothesis-testing system, we predicted little effect of regulatory fit on information integration processing once the procedural-based learning system began to dominate. However, COVIS assumes that early in learning, there is a bias toward the hypothesis-testing system. A regulatory fit is hypothesized to affect processing in the hypothesistesting system by increasing cognitive flexibility. This should have several effects on information integration category learning. First, increased cognitive flexibility should lead regulatory fit participants (i.e., those with a prevention focus) to rely longer on rule-based strategies than do participants in a regulatory mismatch condition. This should be observable early in learning for the prevention participants as a performance decline (i.e., lower accuracy) and in the model fits as a higher proportion of prevention participants' data sets being fit by rule-based models. Second, because increased cognitive flexibility should lead participants to be more likely to abandon the highly salient position dimension in favor of the lower salience conjunctive rules, we should observe a higher proportion of regulatory fit participants using conjunctive rules early in learning, whereas regulatory mismatch participants using rules should be more likely to be using the highly salient position dimension. Finally, any effect of regulatory fit should disappear later in learning, because participants in both regulatory focus conditions are using procedural-based learning strategies or because those who are still using rules are using conjunctive rules.

\section{Method}

\section{Participants}

Forty-one participants from the university community completed the study and received course credit or $\$ 6$ payment for participation. Twenty participated in the prevention focus condition, and 21 in the promotion focus condition. No one participated in more than one condition. In addition, the participants were given the opportunity to obtain a 1-in-10 chance of receiving $\$ 50$ in a random drawing.

\section{Stimuli, Stimulus Generation, and Procedure}

The category distribution parameters are outlined in Table 2 . The procedure used for generating and presenting the stimuli, as well as the general experimental procedure, was identical to that in Experiment 1 .

\section{Results and Theoretical Analyses}

\section{Accuracy Analyses}

As in Experiment 1, we will begin by examining the proportion of participants who met or exceeded the $90 \%$ perfor- 
mance criterion. First, we computed the first block in which the participants met or exceeded the performance criterion. As was predicted from the regulatory fit hypothesis, the promotion participants (after 8.2 blocks, on average) exceeded criterion slightly sooner than did the prevention participants (after 9.1 blocks, on average). Even so, this difference was not significant. In Experiment 1, the participants reached criterion after approximately 4 blocks of trials, and $60 \%$ of the participants reached criterion by the 12th block of trials. In the present experiment, on the other hand, it took the participants, on average, over twice as long to reach criterion, and by the 12 th block of trials, only $30 \%$ of the participants had reached criterion. Taken together, these data indicate that this task was more difficult than the task in Experiment 1.
Figure 6A displays the average accuracy rates for the promotion and prevention participants. As was expected from the regulatory fit hypothesis, the promotion focus participants (who were in a regulatory mismatch condition) were more accurate than were the prevention focus participants (who were in a regulatory fit condition) early in learning (i.e., during Blocks 1-5), but later in learning, the accuracy rates were nearly identical. Specifically, the promotion participants achieved $75 \%$ accuracy during Blocks $1-5$, whereas the prevention participants achieved only $69 \%$ accuracy $[t(39)=2.06, p<.05]$. During Blocks 6-12, on the other hand, the promotion (82\%) and the prevention $(82 \%)$ focus participants achieved the same accuracy rate $(t<1)$.
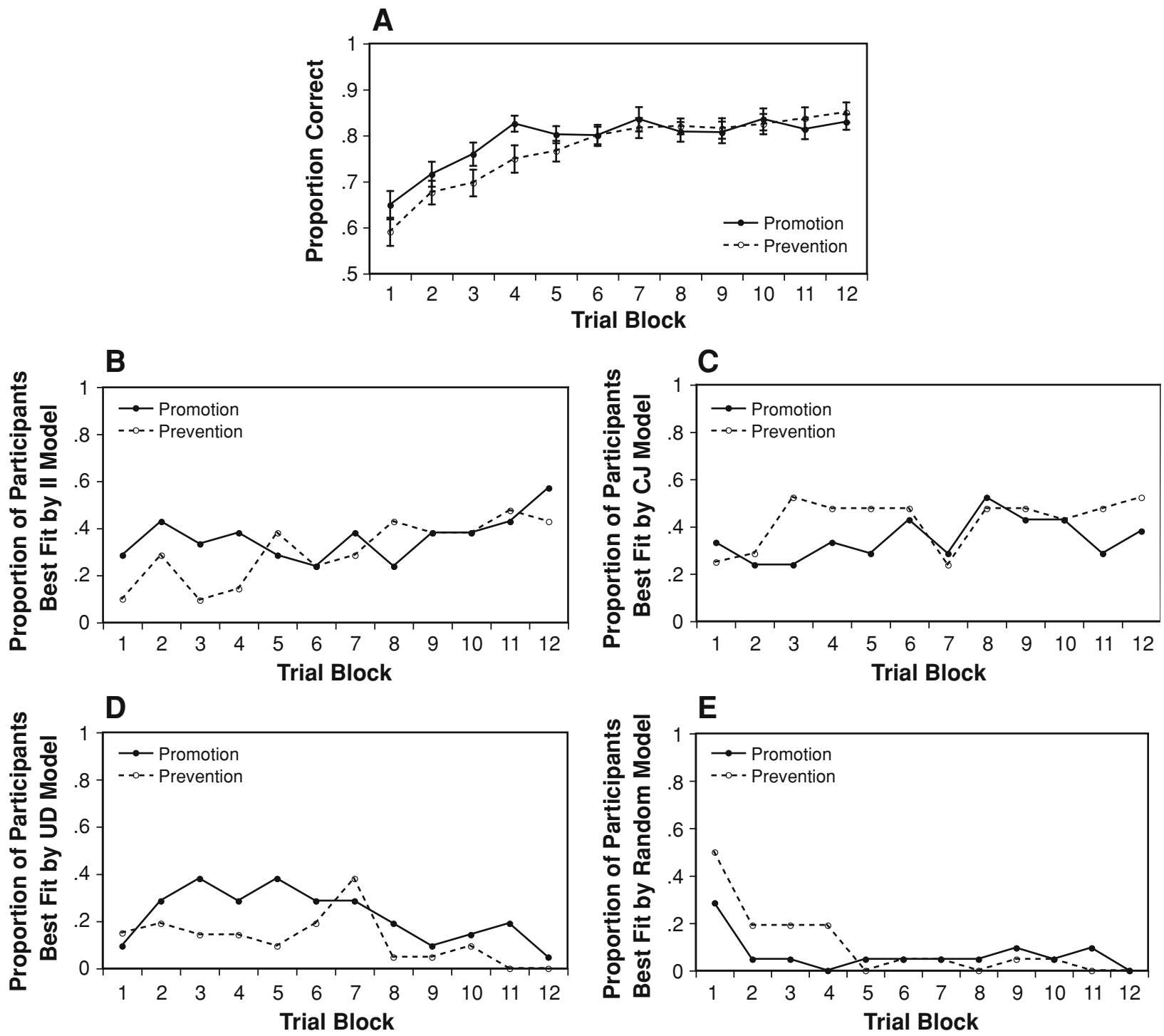

Figure 6. (A) Proportion correct (averaged across observers) in Experiment 3, along with standard error bars. (B) Proportion of participants best fit by an information integration (II) model. (C) Proportion of participants best fit by a conjunctive (CJ) model. (D) Proportion of participants best fit by a unidimensional (UD) model. (E) Proportion of participants best fit by a random responder model. 


\section{Model-Based Analyses}

Several decision bound models were fit separately to each participant's block-by-block data. The three unidimensional-rule-based models described in Experiment 1 were fit to the data (two parameters each). In addition, several conjunctive-rule-based models were fit to the data (three parameters each). These included the suboptimal conjunctive model from Experiment 1 that applied a rule on length and orientation, as well as conjunctive models that applied rules on length and position or orientation and position. A random responder model that assumed a fixed probability of responding "A" for all the stimuli was also applied (one parameter). Three procedural-based learning models were applied. One assumed that the participant used the optimal decision bound displayed in Figure 1B (one parameter), a second estimated the best-fitting slope and intercept of this bound from the data (three parameters), and a third assumed that the decision bound was piecewise linear with two separate linear components (five parameters). As in Experiment 1, we used AIC to determine the best-fitting model for each participant in each block. On average, the best-fitting model from the final block of data accounted for $84 \%$ and $87 \%$ of the responses for the promotion and the prevention participants, respectively, suggesting a good account of the data.

Figures $6 \mathrm{~B}-6 \mathrm{E}$ display the proportions of participants best fit by an information integration, conjunctive rule, unidimensional rule, or random responder model, respectively, for each block of trials. Table 6 displays the percentage of participants best fit by each model class, along with the average accuracy rate during early learning (Blocks 1-5) and late learning (Blocks 6-12). The analyses were aggregated in this way because the accuracy rates and block-by-block model results (see Figure 6) suggested that the effects of regulatory fit were restricted to Blocks $1-5$. Although the data from all three unidimensional-rulebased models are combined, the overwhelming majority of the time (over $80 \%$ ) the unidimensional position model provided the better fit.

Several comments are in order. First, as was expected, the effect of regulatory focus on information integration category learning was restricted to the first several blocks of trials (early learning). The model proportions were quite similar across regulatory focus conditions late in learning. Second, and as was predicted from the regulatory fit hypothesis, during early learning, an information integration model fit a larger proportion of the participants' data in the regulatory mismatch condition $(37 \%)$ than in the regulatory fit condition (20\%). This difference was significant, on the basis of a binomial test, in Blocks 1, 3, and $4(p<.05)$. Third, the proportion of the participants' data best fit by a rule-based model during early learning in the regulatory fit $(60 \%)$ and regulatory mismatch $(63 \%)$ conditions did not differ; however, the proportion of these data sets best fit by a conjunctive model was higher in the fit condition (40\%) than in the mismatch condition $(29 \%)$, although the effect was significant in Block 3 only. By extension, the proportion best fit by a unidimensional model
Table 6

Percentages of Promotion and Prevention Focus Participants for Which an Information Integration, Conjunctive, Unidimensional, or Random Responder Model Provided the Best Account of the Data, Along With the Average Accuracy Rates Obtained for Those Participants During Early (Blocks 1-5) and Late (Blocks 6-12) Learning

\begin{tabular}{|c|c|c|c|c|}
\hline \multirow[b]{2}{*}{ Model } & \multirow[b]{2}{*}{$\begin{array}{l}\text { Situational } \\
\text { Focus }\end{array}$} & \multirow[b]{2}{*}{ Measure } & \multicolumn{2}{|c|}{ Blocks } \\
\hline & & & $\begin{array}{c}\text { Early } \\
\text { Learning }\end{array}$ & $\begin{array}{c}\text { Late } \\
\text { Learning }\end{array}$ \\
\hline \multirow[t]{4}{*}{ II } & Promotion & Model \% & 34 & 37 \\
\hline & & Proportion correct & .77 & .85 \\
\hline & Prevention & Model \% & 20 & 37 \\
\hline & & Proportion correct & .70 & .88 \\
\hline \multirow[t]{4}{*}{ CJ } & Promotion & Model \% & 29 & 39 \\
\hline & & Proportion correct & .79 & .84 \\
\hline & Prevention & Model \% & 40 & 44 \\
\hline & & Proportion correct & .76 & .80 \\
\hline \multirow[t]{4}{*}{ UD } & Promotion & Model \% & 29 & 18 \\
\hline & & Proportion correct & .77 & .82 \\
\hline & Prevention & Model \% & 14 & 11 \\
\hline & & Proportion correct & .73 & .82 \\
\hline \multirow[t]{4}{*}{ Random } & Promotion & Model \% & 9 & 5 \\
\hline & & Proportion correct & .49 & .48 \\
\hline & Prevention & Model \% & 21 & 3 \\
\hline & & Proportion correct & .50 & .58 \\
\hline
\end{tabular}

Note-II, information integration model; CJ, conjunctive models; UD, unidimensional models; Random, random responder model.

was lower in the fit condition (14\%) than in the mismatch condition (29\%). This difference was significant in Blocks 3 and 5. Thus, the participants in a regulatory fit condition were faster to abandon a highly salient unidimensional rule in favor of a more accurate conjunctive-rule-based strategy.

Finally, and somewhat unexpectedly, the proportion of participants whose data were best fit by a random responder model early in learning was moderate in the fit condition (21\%) and was higher than the much lower proportion observed in the mismatch condition (9\%). Although this difference was significant in Blocks $1-4$, the largest proportions of participants were best fit by this model during Block 1 (50\% for regulatory fit participants and $29 \%$ for regulatory mismatch participants). One interpretation of this finding is that participants give up and respond randomly. The fact that few participants' data are well fit by this model after the first few blocks argues against this interpretation. A more likely interpretation is that regulatory fit participants generate and test numerous different rule-based strategies during the first few blocks. Under these conditions, no model that assumes a fixed decision strategy throughout the whole block will provide a good account of the data. Rather, a random responder model that assumes a fixed response probability for all the stimuli will be likely to fit best.

Taken together, the model-based analyses suggest that when cognitive flexibility is disadvantageous, due to the need to learn an information integration category structure, a regulatory fit slows the shift away from rule-based strategies toward information integration strategies. In 
some cases, the increase in cognitive flexibility that results from a regulatory fit leads to frequent changes in strategy that evidence themselves by a good fit of the random responder model. In other cases, the increase in flexibility leads the participant to rapidly abandon highly salient unidimensional strategies in favor of less salient, but more accurate, conjunctive strategies.

\section{Discussion}

Experiment 3 provided the final critical test of our regulatory-fit-cognitive-flexibility hypothesis. An information integration category structure for which cognitive flexibility was disadvantageous was used, along with a losses reward structure. The regulatory fit hypothesis predicts greater cognitive flexibility for the prevention participants and, thus, poorer performance, whereas the promotion focus hypothesis predicts greater cognitive flexibility for the promotion participants and, thus, poorer performance. In support of the regulatory fit hypothesis, the prevention focus participants reached the performance criterion later, obtained lower early learning accuracy rates, and took longer to shift away from rule-based strategies toward information integration strategies.

\section{GENERAL DISCUSSION}

In this article, our aim has been to bridge the gap between research on motivation and cognition by bringing the study of motivational influences back into the realm of cognition. In particular, we examined the effects of regulatory fit (Higgins, 2000; Higgins et al., 2003; Shah et al., 1998 ) on perceptual classification learning. A person is assumed to be in a regulatory fit when the situational regulatory focus matches the reward structure of the task - for example, when a situational promotion focus is paired with a gains reward structure or a situational prevention focus is paired with a losses reward structure. We assumed that a regulatory fit supports more flexible cognitive processing than does a regulatory mismatch. When the perceptual classification task is one for which cognitive flexibility is advantageous, we predict better classification learning for regulatory fit participants. On the other hand, when the perceptual classification task is one for which cognitive flexibility is disadvantageous, we predict better classification learning for regulatory mismatch participants.

We tested this hypothesis across three perceptual classification-learning experiments. In Experiment 1, the participant needed to abandon a simple, unidimensional rule along a salient dimension in favor of a more complex, conjunctive rule along two less salient dimensions. In Experiment 1A, we used a gains reward structure, and in Experiment 1B, a losses reward structure. A qualitative shift in strategy of this sort should be enhanced by greater cognitive flexibility, and thus we predicted better performance under a regulatory fit than under a regulatory mismatch. This hypothesis was supported by the data, with faster, more accurate learning for promotion participants in Experiment 1A and for prevention participants in
Experiment 1B. Model-based analyses suggested that the performance advantage that emerged for the regulatory fit participants in both experiments was due to a faster shift away from simple, highly salient unidimensional rules toward the more complex, lower salience conjunctive rule during the intermediate stage of learning.

The results from Experiment 1 suggest that a regulatory fit leads to increased cognitive flexibility but are also consistent with the simpler hypothesis that a regulatory fit simply leads to better performance. To test this hypothesis, Experiments 2 and 3 were conducted. In Experiment 2, we used a gains reward structure and a rule-based classification task for which cognitive flexibility was disadvantageous. In Experiment 3, we used a losses reward structure and an information integration task for which cognitive flexibility was disadvantageous. If a regulatory fit leads to increased cognitive flexibility, as we propose, participants in a regulatory fit should perform worse in both experiments than participants in a regulatory mismatch. On the other hand, if a regulatory fit simply leads to better learning, regulatory fit participants should perform better than regulatory mismatch participants, replicating the effect observed in Experiment 1.

In Experiment 2, the two-dimensional nature of the optimal rule was apparent early in learning, and so changing the decision criterion gradually was a better strategy than attempting a qualitative shift in strategy. Under these conditions and when the goal was attainable, we predicted no effect of regulatory fit, because the regulatory fit participants would not choose to make big changes in their strategy and would behave similarly to regulatory mismatch participants. On the other hand, when the goal was unattainable, the regulatory fit participants, who have high levels of cognitive flexibility, would be more willing than low cognitive flexibility, regulatory mismatch participants to try large changes in strategy in an attempt to "find" the correct rule. Because the form of the correct rule was obvious early in learning, these large shifts in strategy would adversely affect regulatory fit participants' performance. Thus, we predicted that performance would be worst for the regulatory participants when the goal was unattainable, with no difference across the two attainable conditions. These predictions were supported by the accuracy data. In addition, and as was predicted, the locus of the promotion-unattainable condition deficit appeared to be in worse memory and more variable placement of the decision criteria.

In Experiment 3, we used a category structure for which cognitive flexibility was disadvantageous. Instead of replicating the category structure used in Experiment 2, we used an information integration category structure that shared many properties (e.g., same three-dimensional stimuli, same optimal accuracy, same bonus criteria) with the category structure used in Experiment 1. Cognitive flexibility was predicted to increase the participants' reliance on rule-based strategies and to slow the shift toward information integration strategies. Because cognitive flexibility was disadvantageous, the regulatory fit hypothesis 
predicted faster learning for the promotion focus participants (who were in a mismatch condition) than for the prevention focus participants (who were in a regulatory fit condition). In line with the regulatory fit hypothesis, the promotion focus participants reached the performance criterion sooner, obtained higher accuracy rates early in learning, and abandoned rules in favor of information integration strategies more quickly than did the prevention focus participants.

Taken together, the results from Experiments 2 and 3, along with the results from Experiment 1, support the regulatory fit hypothesis over the simpler hypothesis that a regulatory fit leads to better overall learning regardless of whether cognitive flexibility is advantageous or disadvantageous.

\section{Implications for Research on Cognition}

These data suggest that motivational focus can be manipulated easily within the framework of cognitive research and that, at least within the domain of classification learning, this simple manipulation can have a large and easily interpretable effect on performance. Our hope is that this work will stimulate other researchers to begin to examine the effects of motivation on cognition. One reason why it is important to explore the influence of regulatory focus on cognitive processing is that many studies in cognitive psychology have an implicit promotion focus that goes unrecognized. For example, in many studies, experimental participants have received course credit or a monetary payment that often has been accompanied by a bonus for good performance. In other cases, participants have been told to complete a task and that they would be allowed to leave as soon as they had achieved a certain performance criterion. Both of these situations induce a mild promotion focus. Although researchers generally have used these procedures in an attempt to "motivate" participants to perform well, motivation has been ill defined. In addition, most studies in cognitive psychology have utilized a gains reward structure that has biased participants toward maximizing some measure, such as accuracy. Thus, the majority of studies have placed participants in a regulatory fit. As the present data suggest, this may be advantageous when flexibility in the task is beneficial but may have adverse performance consequences when flexibility in the task is not beneficial. Thus, the bulk of our data in cognitive psychology may be drawn from conditions in which the participants have had a regulatory fit.

This work also has implications for observed asymmetries between gains and losses in decision making (e.g., Kahneman \& Tversky, 1979). The present experiments suggest that the influence of gains or losses on performance depends both on people's regulatory focus and on the nature of the task being carried out. Both gains and losses can lead to cognitive flexibility when there is a regulatory fit. This work extends research by Shah and Higgins (1997) that suggested that asymmetries between gains and losses observed in previous research may reflect (in part) the fact that this work has not focused explicitly on motivational factors.

\section{Decision Criterion Learning and Regulatory Fit}

We recently tested the regulatory fit hypothesis in the domain of decision criterion learning (Markman et al., 2005). Participants learned a noisy decision criterion under one of four conditions constructed from the factorial combination of situational regulatory focus (promotion or prevention) with the reward structure of the task (gains or losses). In this study, we tested a different form of cognitive flexibility by manipulating the nature of the payoffs associated with correct and incorrect responses. In the gains condition, the participants earned more points for being correct than for making an error and earned more points for being correct for Category A than for Category B. In the losses condition, the participants lost fewer points for being correct than for making an error and lost fewer points for being correct for Category A than for Category B. When the payoffs are asymmetric in this way, the decision criterion that maximizes reward is shifted away from the decision criterion that maximizes accuracy. Across numerous studies (reviewed in Maddox, 2002), it has been shown that participants have a bias toward accuracy maximization. We hypothesized that cognitive flexibility was associated with a greater likelihood of abandoning the accuracy-maximizing criterion in favor of the reward-maximizing criterion. In support of this prediction, the decision criterion estimates from regulatory fit participants (i.e., promotion focus participants in a gains reward structure and prevention focus participants in a losses reward structure) were closer to the optimal reward-maximizing decision criterion than were the decision criterion estimates from regulatory mismatch participants (i.e., promotion focus participants in a losses reward structure and prevention focus participants in a gains reward structure). These results, along with those from the present study, suggest that the hypothesis that a regulatory fit leads to increased cognitive flexibility is robust and generalizes across perceptual classification and decisionmaking tasks. Future work should continue to extend the regulatory fit hypothesis to other empirical domains.

\section{Summary}

This article has reported the results from three perceptual classification experiments that provided a critical test of Maddox et al.'s (2006) prediction that regulatory fit leads to cognitive flexibility. On this view, there is a complex three-way interaction underlying cognitive performance. A person's regulatory focus at a given time interacts with the reward structure of the task to produce either a regulatory fit or a mismatch. Regulatory fit leads to greater cognitive flexibility than does regulatory mismatch. Whether a regulatory fit leads to good performance in a task, however, depends on whether flexibility is required to solve the task correctly.

In support of this view, the participants in Experiment 1 with a regulatory fit performed much better than did those with a regulatory mismatch in a complex perceptual classification task that required abandoning a simple unidimensional rule in favor of a more complex conjunctive rule. Further support for this fit-flexibility hypothesis came from 
Experiments 2 and 3, in which the participants with a regulatory mismatch performed better than those with a regulatory fit on classification tasks that required a more gradual and incremental learning process. The fit-flexibility view is supported both by overall accuracy of classification and by detailed patterns of strategy change that emerge from model-based analyses. Thus, this work demonstrates the power of exploring complex hypotheses about motivational influences on cognition with modeling techniques that support the analysis of performance changes in individual participants over the course of a study.

\section{REFERENCES}

Aarts, H., Gollwitzer, P. M., \& Hassin, R. R. (2004). Goal contagion: Perceiving is for pursuing. Journal of Personality \& Social Psychology, 87, 23-37.

AKAIKE, H. (1974). A new look at the statistical model identification. Transactions on Automatic Control, 19, 716-723.

Amodio, D. M., Shah, J. Y., Sigelman, J., Brazy, P. C., \& HarmonJONES, E. (2004). Implicit regulatory focus associated with asymmetrical frontal cortical activity. Journal of Experimental Social Psychology, 40, 225-232.

Ashby, F. G. (1992). Multidimensional models of categorization. In F. G. Ashby (Ed.), Multidimensional models of perception and cognition (pp. 449-483). Hillsdale, NJ: Erlbaum.

Ashby, F. G., Alfonso-Reese, L. A., Turken, A. U., \& Waldron, E. M. (1998). A neuropsychological theory of multiple systems in category learning. Psychological Review, 105, 442-481.

Ashby, F. G., Isen, A. M., \& TuRKEN, A. U. (1999). A neuropsychological theory of positive affect and its influence on cognition. Psychological Review, 106, 529-550.

AsHBY, F. G., \& MADDOX, W. T. (1993). Relations between prototype, exemplar, and decision bound models of categorization. Journal of Mathematical Psychology, 37, 372-400.

Ashby, F. G., \& Maddox, W. T. (2005). Human category learning. Annual Review of Psychology, 56, 149-178.

AshBY, F. G., MADDOX, W. T., \& LEE, W. W. (1994). On the dangers of averaging across subjects when using multidimensional scaling or the similarity-choice model. Psychological Science, 5, 144-151.

Ashby, F. G., \& Valentin, V. V. (2005). The theory of perceptual category learning. In H. Cohen \& C. Lefebvre (Eds.), Handbook of categorization in cognitive science (pp. 560-572). New York: Elsevier.

Ashby, F. G., \& Waldron, E. M. (1999). On the nature of implicit categorization. Psychonomic Bulletin \& Review, 6, 363-378.

Avnet, T., \& HigGINS, E. T. (2003). Locomotion, assessment, and regulatory fit: Value transfer from "how" to "what." Journal of Experimental Social Psychology, 39, 525-530.

CARver, C. S., \& Scheier, M. F. (1998). On the self-regulation of behavior. New York: Cambridge University Press.

Crowe, E., \& Higgins, E. T. (1997). Regulatory focus and strategic inclinations: Promotion and prevention in decision-making. Organizational Behavior \& Human Decision Processes, 69, 117-132.

CunninghaM, W. A., RaYe, C. L., \& Johnson, M. K. (2005). Neural correlates of evaluation associated with promotion and prevention regulatory focus. Cognitive, Affective, \& Behavioral Neuroscience, 5, 202-211.

ERICKSON, M. A., \& KruschKe, J. K. (1998). Rules and exemplars in category learning. Journal of Experimental Psychology: General, 127, 107-140.

EsTES, W. K. (1956). The problem of inference from curves based on group data. Psychological Bulletin, 53, 134-140.

Ferguson, M. J., \& BARGH, J. A. (2004). Liking is for doing: The effects of goal pursuit on automatic evaluation. Journal of Personality \& Social Psychology, 87, 557-572.

FishbaCh, A., Friedman, R. S., \& KRUgLAnSKI, A. W. (2003). Leading us not unto temptation: Momentary allurements elicit overriding goal activation. Journal of Personality \& Social Psychology, 84, 296-309.

Gray, J. A. (1982). The neuropsychology of anxiety: An enquiry into the functions of the septo-hippocampal system. Oxford: Oxford University Press.

HigGINS, E. T. (1987). Self-discrepancy: A theory relating self and affect. Psychological Review, 94, 319-340.

HigGins, E. T. (1997). Beyond pleasure and pain. American Psychologist, 52, 1280-1300.

HiGGINS, E. T. (2000). Making a good decision: Value from fit. American Psychologist, 55, 1217-1230.

Higgins, E. T., Chen Idson, L., Freitas, A. L., Spiegel, S., \& Molden, D. C. (2003). Transfer of value from fit. Journal of Personality \& Social Psychology, 84, 1140-1153.

KAHNEMAN, D., \& TVERSKY, A. (1979). Prospect theory: An analysis of decision under risk. Econometrica, 47, 263-291.

KÉRI, S. (2003). The cognitive neuroscience of category learning. Brain Research Reviews, 43, 85-109.

LEE, A. Y., \& AAKER, J. L. (2004). Bringing the frame into focus: The influence of regulatory fit on processing fluency and persuasion. Journal of Personality \& Social Psychology, 86, 205-218.

LEWIN, K. (1935). A dynamic theory of personality. New York: McGrawHill.

MadDox, W. T. (1999). On the dangers of averaging across observers when comparing decision bound models and generalized context models of categorization. Perception \& Psychophysics, 61, 354-375.

MAdDOX, W. T. (2002). Toward a unified theory of decision criterion learning in perceptual categorization. Journal of the Experimental Analysis of Behavior, 78, 567-595.

MADDOX, W. T., \& AshBY, F. G. (1993). Comparing decision bound and exemplar models of categorization. Perception \& Psychophysics, 53, 49-70.

MADDOX, W. T., \& Ashby, F. G. (2004). Dissociating explicit and procedural-learning based systems of perceptual category learning. Behavioural Processes, 66, 309-332.

MADDOX, W. T., \& EsTES, W. K. (2004). Predicting true patterns of cognitive performance from noisy data. Psychonomic Bulletin \& Review, 11, 1129-1135.

Maddox, W. T., Markman, A. B., \& Baldwin, G. C. (2006). Using classification to understand the motivation-learning interface. In B. H. Ross \& A. B. Markman (Eds.), The psychology of learning and motivation (Vol. 47, pp. 213-249). New York: Elsevier.

Markman, A. B., Baldwin, G. C., \& Maddox, W. T. (2005). The interaction of payoff structure and regulatory focus in classification. Psychological Science, 16, 852-855.

MARKMAN, A. B., \& BRENDL, C. M. (1999). The influence of goals on value and choice. In D. L. Medin (Ed.), The psychology of learning and motivation (Vol. 39, pp. 97-129). San Diego: Academic Press.

Markman, A. B., Maddox, W. T., Worthy, D. A., \& Baldwin, G. C. (in press). Using regulatory focus to explore implicit and explicit processing. Journal of Consciousness Studies.

Mednick, M. T., Mednick, S. A., \& Mednick, E. V. (1964). Incubation of creative performance and specific associative priming. Journal of Abnormal \& Social Psychology, 69, 84-88.

Miller, N. E. (1959). Liberalization of basic S-R concepts: Extensions to conflict behavior, motivation, and social learning. In S. Koch (Ed.), Psychology: A study of a science. General and systematic formulations, learning, and special processes (pp. 196-292). New York: McGraw-Hill.

MyUnG, I. J. (2000). The importance of complexity in model selection. Journal of Mathematical Psychology, 44, 190-204.

PitT, M. A., MyUng, I. J., \& ZhANG, S. (2002). Toward a method of selecting among computational models of cognition. Psychological Review, 109, 472-491.

PoldRACK, R. A., \& PACKard, M. G. (2003). Competition among multiple memory systems: Converging evidence from animal and human brain studies. Neuropsychologia, 41, 245-251.

SCHACTER, D. L. (1987). Implicit memory: History and current status. Journal of Experimental Psychology: Learning, Memory, \& Cognition, 13, 501-518.

SHAH, J., \& HigGINS, E. T. (1997). Expectancy $\times$ value effects: Regulatory focus as determinant of magnitude and direction. Journal of Personality \& Social Psychology, 73, 447-458.

ShAH, J., Higgins, E. T., \& FriedMAN, R. S. (1998). Performance incen- 
tives and means: How regulatory focus influences goal attainment Journal of Personality \& Social Psychology, 74, 285-293.

SMITH, J. D., \& Minda, J. P. (1998). Prototypes in the mist: The early epochs of category learning. Journal of Experimental Psychology: Learning, Memory, \& Cognition, 24, 1411-1436.

SQUIRE, L. R. (1992). Memory and the hippocampus: A synthesis from findings with rats, monkeys and humans. Psychological Review, 99. 195-231.

TaKANE, Y., \& Shibayama, T. (1992). Structure in stimulus identification data. Hillsdale, NJ: Erlbaum.

Wickens, T. D. (1982). Models for behavior: Stochastic processes in psychology. San Francisco: Freeman.

\section{NOTES}

1. Two factors control the regulatory focus of an individual at any given moment. First, individuals can have a chronically active focus that is affected by whether they are predisposed toward achieving ideal states (associated with a chronic promotion focus), which are defined as that person's hopes, desires, or aspirations, or toward responsibilities (associated with a chronic prevention focus), which are defined as that person's duties, obligations, or responsibilities. Second, individuals can have a regulatory focus induced by situational factors. Pursuing a particular gain in the environment can activate a situational promotion focus, and avoiding a particular loss can activate a situational prevention focus. In our experiments, we explicitly manipulated situational regulatory focus in order to overcome potential individual differences in participants' chronically active focus.
2. An alternative approach is to compare frequencies using a $\chi^{2}$ test. The final block frequency difference was significant with a $\chi^{2}$ test $\left[\chi^{2}(1)=\right.$ $4.267, p<.05]$. However, due to the lower power associated with $\chi^{2}$, the only other frequency differences that emerged were in Blocks 3, 5, and 10.

3. We also fit a random responder model that assumed a fixed probability of responding "A" for all the stimuli. This model never provided the best fit for more than 1 participant in any block. In light of this fact, we excluded this model from our analyses.

4. We collapsed across optimal and suboptimal conjunctive models because the proportion of participants who were optimal was low, although accuracy for the optimal participants was higher. Similarly, we collapsed across the unidimensional length and orientation models because the proportion of participants was low for both models and performance was similar.

5. An alternative approach is to compare frequency counts of conjunctive versus nonconjunctive rule models, using a $\chi^{2}$ test. This test is less powerful and yielded significant difference only in Blocks 5 and 8 .

6. Information integration models (see Maddox \& Ashby, 2004, for details) and the random responder model were also applied to the data, but these rarely provided the best account of the data and will not be discussed further.

(Manuscript received April 21, 2005; revision accepted for publication July 25,2005 .) 